Research Article

\title{
Connectivity Indices of Intuitionistic Fuzzy Graphs and Their Applications in Internet Routing and Transport Network Flow
}

\author{
Tulat Naeem, ${ }^{1}$ Abdu Gumaei $\left(\mathbb{D},{ }^{2}\right.$ Muhammad Kamran Jamil $\mathbb{D}^{1},{ }^{1}$ Ahmed Alsanad $\left(\mathbb{D},{ }^{3}\right.$ \\ and Kifayat Ullah ${ }^{1}{ }^{1}$ \\ ${ }^{1}$ Department of Mathematics, Riphah Institute of Computing and Applied Sciences, Riphah International University, \\ Lahore, Pakistan \\ ${ }^{2}$ Computer Science Department, Faculty of Applied Sciences, Taiz University, Taiz 6803, Yemen \\ ${ }^{3}$ STC's Artificial Intelligence Chair, Department of Information Systems, College of Computer and Information Sciences, \\ King Saud University, Riyadh 11451, Saudi Arabia
}

\begin{abstract}
Correspondence should be addressed to Abdu Gumaei; abdugumaei@gmail.com, Ahmed Alsanad; aasanad@ksu.edu.sa, and Kifayat Ullah; kifayat.khan.dr@gmail.com
\end{abstract}

Received 11 May 2021; Accepted 30 July 2021; Published 14 August 2021

Academic Editor: G. Muhiuddin

Copyright (c) 2021 Tulat Naeem et al. This is an open access article distributed under the Creative Commons Attribution License, which permits unrestricted use, distribution, and reproduction in any medium, provided the original work is properly cited.

Connectivity index $(\mathscr{C I})$ has a vital role in real-world problems especially in Internet routing and transport network flow. Intuitionistic fuzzy graphs (IFGs) allow to describe two aspects of information using membership and nonmembership degrees under uncertainties. Keeping in view the importance of $\mathscr{C} \mathscr{I} s$ in real life problems and comprehension of IFGs, we aim to develop some $\mathscr{C} \mathscr{I} s$ in the environment of IFGs. We introduce two types of $\mathscr{C} \mathscr{F} S$, namely, $\mathscr{C} \mathscr{I}$ and average $\mathscr{C} \mathscr{F}$, in the frame of IFGs. In spite of that, certain kinds of nodes called IF connectivity enhancing node (IFCEN), IF connectivity reducing node (IFCRN), and IF neutral node are introduced for IFGs. We have introduced strongest strong cycles, $\theta$-evaluation of vertices, cycle connectivity, and $\mathscr{C} \mathscr{I}$ of strong cycle. Applications of the $\mathscr{C} \mathscr{I} S$ in two different types of networks are done, Internet routing and transport network flow, followed by examples to show the applicability of the proposed work.

\section{Introduction}

Zadeh [1] presented the idea of fuzzy set (FS) by giving membership grades to the objects of a set ranging from zero to one. Many concepts of crisp set theory like inclusion, union, intersection, complement, etc., were established for FSs. FS theory opened the way to fuzzy logic and fuzzy control systems. In the beginning, probability theory was the only tool to handle problems of uncertainty, facing science, technology, and real life problems. FS theory has many applications in different areas such as inventory control model [2], decision-making problems [3], and intelligence science [4]. More applications can be found in $[5,6]$. A recent research to treat COVID-19 disease with FS approach is given in [7].

In 1975, Rosenfeld [8] studied fuzzy graphs (FGs). After that, Yeh and Bang [9] presented the same concept independently during the same period. Rosenfeld defined some basic properties of fuzzy relations including fuzzy bridges and trees with their properties, while Yeh and Bang gave the concept of connectedness of FGs along with applications. Mordeson [10] proposed the work for fuzzy line graphs. Massa'deh $[11,12]$ introduced complete FGs, regular FGs, complement of FGs, and some other properties. Mathew and Sunitha introduced types of arcs such as $\alpha$-strong, $\beta$-strong, and $\delta$-arcs in FGs [13]. The authors gave various concepts like strong arcs in [14], fuzzy end nodes in [15], and geodesic in [16]. Recently, Akram presented concepts like bipolar FGs in [17] and energy of bipolar FGs in [18]. Jan et al. studied the concept of cubic bipolar FGs with an application in a social network [19]. FGs are useful in representing relationships under uncertainty. FGs are used in various areas like human trafficking, disaster management system, decision-making method, etc. 
The extension of FS is intuitionistic fuzzy set (IFS) presented by Atanassov [20] in 1986. He added a new component in the definition of FS, which is known as the degree of nonmembership or falsity degree. IFS is the generalization of FS with the requirement that the sum of both degrees cannot exceed 1. Many researchers have applied IFSs in decision-making problems. Chen [21] proposed to measure the degree of similarity between vague sets. Similarity measures for discrete, as well as for continuous, sets are given in [22] and applied in pattern recognition problems. More work on IFSs can be found in [23-25]. Fields of applications of IFSs are Computer Science, Engineering, Medicine, Chemistry, Economics, etc.

The generalization of FG is intuitionistic fuzzy graph (IFGs) explained elaborately by Parvathi and Karunambigai [26]. They also gave the concepts of path, bridge, and cut vertices in IFGs. Dhavudh and Srinivasan [27] defined IFG of second kind, and IFG of nth type was developed by Davvaz et al. [28]. Karunambigai and Buvaneswari [29] introduced arcs in IFGs like strong arcs, weakest arcs, strong path, $\alpha$-strong, $\beta$-strong, and $\delta$-weak arcs. Karunambigai and kalaivani [30] presented IFGs as the matrix representation. Mishra and $\mathrm{Pal}[31,32]$ discussed the product of two interval valued IFGs, their properties, and regular interval valued IFGs in 2013 and 2017. Fallatah et al. [33] and Alanser et al. [34] introduced new concepts as IF soft graphs and bipolar IFGs. Akram and Alshehri [35] introduced IF cycles and trees. Some misconceptions in the definitions of several generalizations of IFGs are corrected by Jan et al. [36]. IFGs are applied in different areas such as cellular network and decision support systems [37, 38].

Connectivity is the most fundamental and normal parameter related to a network. The stability of a network depends on its connectivity. Binu et al. introduced two measures on connectivity, namely, cyclic $\mathscr{C} \mathscr{I}$ and average cyclic $\mathscr{C} \mathscr{I}$ of FGs [39]. Poulik and Ghorai brought the concept of $\mathscr{C} \mathscr{I}$, average $\mathscr{C} \mathscr{I}$, and types of connectivity nodes under bipolar fuzzy graph environment with applications [40]. Mathew and Mordeson [41] introduced $\mathscr{C} \mathscr{I}$ and $\mathscr{A} \mathscr{C} \mathscr{I}$, studied their properties, and investigated their applications. Binu et al. discussed the concept of Wiener index and relationship between Wiener index and connectivity index with an application to illegal immigration networks [42]. FGs describe only one type of opinion, that is, membership degree, while IFGs describe two types of opinions with the help of membership and nonmembership degrees.

In our paper, we have considered IFG and discussed certain concepts related to IFG. In Section 2, preliminary requirements are given for the work of this paper. In Section 3, we have extended concepts of $\mathscr{C} \mathscr{I}$ and bounds of $\mathscr{C} \mathscr{I}$ for IFG. Section 4 provides $\mathscr{C} \mathscr{I}$ of vertex and edge deleted IF subgraphs. Section 5 presents concepts of the strongest strong cycles, $\theta$-evaluation of vertices, cycle connectivity (CC), and $\mathscr{C} \mathscr{I}$ of strong cycle for IFG. Section 6 deals with $\mathscr{A} \mathscr{C} \mathscr{I}$ along with its properties. Applications of $\mathscr{C} \mathscr{I} s$ are discussed in Section 7. Finally Section 8 concludes this study.

\section{Preliminaries}

Throughout this section, definitions and examples are presented to recall concepts related to IFG, arcs in IFG, and IF-cycles relevant to the present work. Most of the definitions in preliminaries are taken from $[29,35,43]$.

The notion of IFG was proposed by Akram and Davvaz [43] and given as follows:

Definition 1 (see [43]). An IFG is a pair $G=(N, M)$ such that

(1) $V=\left\{u_{1}, u_{2}, u_{3}, \ldots, u_{n}\right\}$ with $T_{N}: V \longrightarrow[0,1]$ and $F_{N}: V \longrightarrow[0,1]$ representing the truth-membership degree and falsity-membership degree of the vertex $u_{i} \in V$ and $0 \leq T_{N}\left(u_{i}\right)+F_{N}\left(u_{i}\right) \leq 1$ for each $u_{i} \in V(0 \leq i \leq n)$.

(2) $E \subseteq V \times V \quad$ with $\quad T_{M}: E \longrightarrow[0,1] \quad$ and $F_{M}: E \longrightarrow[0,1]$ being as follows:

$$
\begin{aligned}
& T_{M}\left(u_{i}, u_{j}\right) \leq \min \left\{T_{N}\left(u_{i}\right), T_{N}\left(u_{j}\right)\right\}, \\
& F_{M}\left(u_{i}, u_{j}\right) \geq \max \left\{F_{N}\left(u_{i}\right), F_{N}\left(u_{j}\right)\right\},
\end{aligned}
$$

and $0 \leq T_{M}\left(u_{i}, u_{j}\right)+F_{M}\left(u_{i}, u_{j}\right) \leq 1$ for every edge $\left(u_{i}, u_{j}\right) \in E$.

The next definition is related to complete IFG given by Parvathi et al. [29].

Definition 2 (see [29]). An IFG $G$ is complete if $T_{M}\left(u_{i}, u_{j}\right)=$ $\min \left\{T_{N}\left(u_{i}\right), T_{N}\left(u_{j}\right)\right\}$ and $F_{M}\left(u_{i}, u_{j}\right)=\max \left\{F_{N}\left(u_{i}\right), F_{N}\right.$ $\left.\left(u_{j}\right)\right\}$ for each $\left(u_{i}, u_{j}\right) \in E$.

The role of path is extremely famous and important in IFGs. The following definition gives us the concept of path in IFGs.

Definition 3. [29] A sequence $u_{1}, u_{2}, u_{3}, \ldots, u_{n}$ of distinct vertices is a path $P$ in an IFG, provided it has one of the conditions given below for some $i$ and $j$.

(1) $T_{M}\left(u_{i}, u_{j}\right)>0$ and $F_{M}\left(u_{i}, u_{j}\right)=0$

(2) $T_{M}\left(u_{i}, u_{j}\right)=0$ and $F_{M}\left(u_{i}, u_{j}\right)>0$

(3) $T_{M}\left(u_{i}, u_{j}\right)>0$ and $F_{M}\left(u_{i}, u_{j}\right)>0$

The strength of paths plays a significant role in IFG settings. The following definition gives us component-wise and whole strength of paths in IFGs.

Definition 4 (see [29]). Let $P=u_{1}, u_{2}, u_{3}, \ldots, u_{n}$ be a path in an IFG. Then,

(1) The T-strength of $P$ is denoted and defined by $S_{T}=$ $\min \left\{T_{M}\left(u_{i}, u_{j}\right)\right\} \forall i, j$

(2) The F-strength of $P$ is denoted and defined by $S_{F}=$ $\max \left\{F_{M}\left(u_{i}, u_{j}\right)\right\} \forall i, j$

(3) $S_{P}=\left(S_{T}, S_{F}\right)$ is called the strength $P$ if both $S_{T}$ and $S_{F}$ exist for the same edge 
The highly connected nodes have significant role to a network. The next definition is about the strength of connectedness between the nodes.

Definition 5 (see [29]). The T-strength of connectedness between two vertices $u_{i}$ and $u_{j}$ is defined by $\operatorname{CONN}_{T(G)}\left(u_{i}, u_{j}\right)=\max \left\{S_{T}\right\}$ and F-strength of connectedness between $u_{i}$ and $u_{j}$ is defined by $\operatorname{CONN}_{F(G)}\left(u_{i}, u_{j}\right)=$ $\min \left\{S_{F}\right\}$ for all possible paths between $u_{i}$ and $u_{j}$,

where $\operatorname{CONN}_{T(G)-\left(u_{i}, u_{j}\right)}\left(u_{i}, u_{j}\right), \operatorname{CONN}_{F(G)-\left(u_{i}, u_{j}\right)}\left(v_{i}, v_{j}\right)$ denote the T-strength of connectedness and F-strength of connectedness between $u_{i}$ and $u_{j}$ attained by removing the edge $\left(u_{i}, u_{j}\right)$ from $G$, respectively.

The following definition gives us the idea of a bridge whose deletion from an IFG increase its number of connected components.

Definition 6 (see [29]). An edge $\left(v_{i}, v_{j}\right)$ is called a bridge in $G$ if either

$$
\begin{aligned}
& \operatorname{CONN}_{T(G)-\left(u_{i}, u_{j}\right)}\left(u_{i}, u_{j}\right)<\operatorname{CONN}_{T(G)}\left(u_{i}, u_{j}\right), \\
& \operatorname{CONN}_{F(G)-\left(u_{i}, u_{j}\right)}\left(u_{i}, u_{j}\right) \geq \operatorname{CONN}_{F(G)}\left(u_{i}, u_{j}\right) \\
& \operatorname{orCONN}_{T(G)-\left(u_{i}, u_{j}\right)}\left(u_{i}, u_{j}\right) \leq \operatorname{CONN}_{T(G)}\left(u_{i}, u_{j}\right) \text {, } \\
& \operatorname{CONN}_{F(G)-\left(u_{i}, u_{j}\right)}\left(u_{i}, u_{j}\right)>\operatorname{CONN}_{F(G)}\left(u_{i}, u_{j}\right) \text {, } \\
& \text { for some } u_{i}, u_{j} \in V(G) \text {. }
\end{aligned}
$$

In other words, deletion of $\left(u_{i}, u_{j}\right)$ reduces the strength of connectedness between any pair of vertices.

The concept of strong and weakest edges is of much importance in IFGs as well as in our study. The next definition is related to the notions of strong and weakest edges.

Definition 7 (see [29]). An edge $\left(u_{i}, u_{j}\right)$ in an IFG is

(1) Strong if $T_{M}\left(u_{i}, u_{j}\right) \geq \operatorname{CONN}_{T(G)}\left(u_{i}, u_{j}\right)$ and $F_{M}\left(u_{i}, u_{j}\right) \leq \operatorname{CONN}_{F(G)}\left(u_{i}, u_{j}\right)$ for each $u_{i}, u_{j} \in V$

(2) Weakest if $T_{M}\left(u_{i}, u_{j}\right)<\operatorname{CONN}_{T(G)}\left(u_{i}, u_{j}\right)$ and $F_{M}\left(u_{i}, u_{j}\right)>\operatorname{CONN}_{F(G)}\left(u_{i}, u_{j}\right)$ for each $u_{i}, u_{j} \in V$

The coming definition gives us the strongest paths between two vertices. This definition is relevant to our work.

Definition 8 (see [29]). A strongest path between two vertices in an IFG $G$ is a path $P$ having its strength equal to $\operatorname{CONN}_{T(G)}\left(u_{i}, u_{j}\right)$ and $\operatorname{CONN}_{F(G)}\left(u_{i}, u_{j}\right)$ lying in the same edge. path.

The following definition tells us the concept of strong

Definition 9 (see [29]). Let $G=(N, M)$ be an IFG. A path $P: u_{i}-u_{j}$ in $G$ is called strong path if $P$ consists of only strong edges.

Example 1. In Figure 1, $T_{M}\left(v_{1}, v_{2}\right)=0.6=\mathrm{CONN}_{T(G)}$ $\left(v_{1}, v_{2}\right)$ and $F_{M}\left(v_{1}, v_{2}\right)=0.6=\operatorname{CONN}_{F(G)}\left(v_{1}, v_{2}\right)$, which implies that $\left(v_{1}, v_{2}\right)$ is a strong arc. Similarly, $\left(v_{2}, v_{3}\right)$,

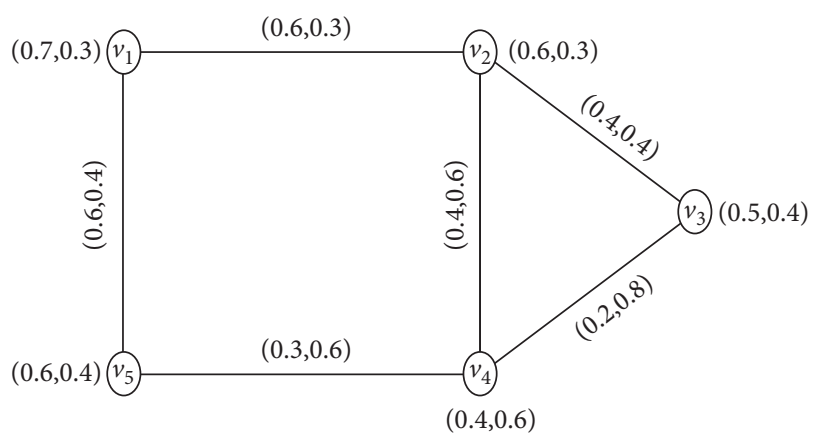

Figure 1: An IFG with strong and weakest arcs.

$\left(v_{1}, v_{5}\right),\left(v_{2}, v_{4}\right)$ are strong arcs and $\left(v_{3}, v_{4}\right),\left(v_{4}, v_{5}\right)$ are weakest arcs. Here, $P=v_{1} v_{2} v_{3}$ is a strong path. In fact, it is the strongest path.

The next definition provides us types of strong arcs in IFG.

Definition 10 (see [29]). An $\operatorname{arc}\left(u_{i}, u_{j}\right)$ in an IFG $G=(N, M)$ is

(1) Called $\quad \alpha$-strong if $\quad T_{M}\left(u_{i}, u_{j}\right)>\operatorname{CONN}_{T}$ $(G)-\left(u_{i}, u_{j}\right) \quad\left(u_{i}, u_{j}\right)$ and $F_{M}\left(u_{i}, u_{j}\right)<\operatorname{CONN}_{F}$ $(G)-\left(u_{i}, u_{j}\right)\left(u_{i}, u_{j}\right)$

(2) Called $\beta$-strong if $T_{M}\left(u_{i}, u_{j}\right)=\operatorname{CONN}_{T(G)-\left(u_{i}, u_{j}\right)}$ $\left(u_{i}, u_{j}\right)$ and $F_{M}\left(u_{i}, u_{j}\right)=\operatorname{CONN}_{F(G)-\left(u_{i}, u_{j}\right)}\left(u_{i}, u_{j}\right)$

(3) Called $\delta$-weak if $T_{M}\left(u, u_{j}\right)<\operatorname{CONN}_{T(G)-\left(u_{i}, u_{j}\right)}$ $\left(u_{i}, u_{j}\right)$ and $F_{M}\left(u_{i}, u_{j}\right)>\operatorname{CONN}_{F(G)-\left(u_{i}, u_{j}\right)}\left(u_{i}, u_{j}\right)$

Example 2. In Figure 2, the arcs $\left(v_{1}, v_{4}\right),\left(v_{2}, v_{3}\right),\left(v_{4}, v_{5}\right),\left(v_{1}, v_{3}\right)$ are $\alpha$-strong, $\left(v_{3}, v_{4}\right)$ is $\beta$-strong, and $\left(v_{1}, v_{2}\right),\left(v_{3}, v_{5}\right)$ are $\delta$-weak.

The following definition gives us different types of strong paths in IFGs.

Definition 11 (see [29]). A path in an IFG containing only $\alpha$-strong arcs is called $\alpha$-strong and a path having only $\beta$-strong arcs is called $\beta$-strong.

The concept of a cycle has a vital role in IFGs. The following definition gives us the concept of a cycle in IFGs environment.

Definition 12 (see [35]).

(1) $G=(N, M)$ is called a cycle if $G^{*}=\left(N^{*}, M^{*}\right)$ is cycle

(2) $G$ is called an IF cycle if $G^{*}=\left(N^{*}, M^{*}\right)$ is cycle and $\nexists$ unique $(x, y) \in M^{*}$ such that

$$
\begin{aligned}
& T_{M}(x, y)=\min \left\{T_{M}(a, b):(a, b) \in M^{*}\right\}, \\
& F_{M}(x, y)=\max \left\{F_{M}(a, b):(a, b) \in M^{*}\right\} .
\end{aligned}
$$

Example 3. In this example, we take $\left(T_{N}(u)\right.$, $\left.F_{N}(u)\right)=(0.3,0.2)$ for all $u \in N^{*}$. Here, $\min \left\{T_{M}(u, v)\right\}=$ 0.2 and $\max \left\{F_{M}(u, v)\right\}=0.3$. Clearly, $G=(N, M)$ is an IF cycle. The graph is shown in Figure 3. 


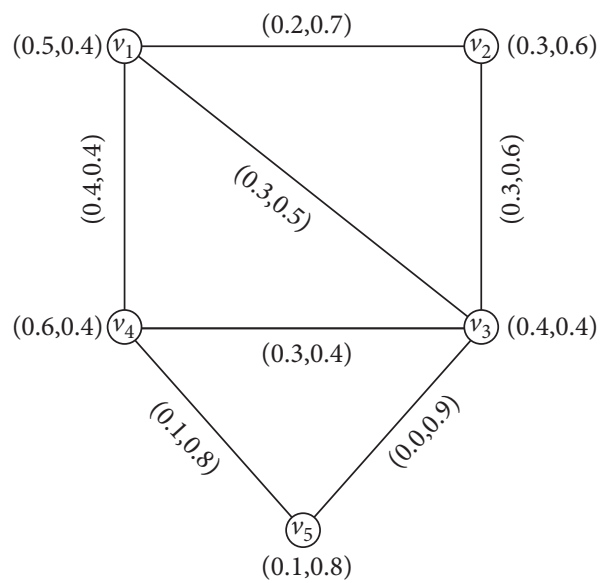

Figure 2: An IFG with $\alpha$-strong, $\beta$-strong, and $\delta$-weak arcs.

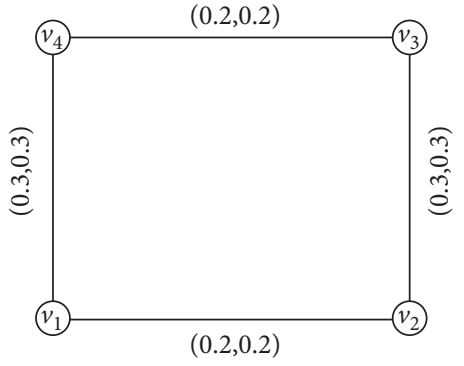

Figure 3: An IF cycle.

The main goal of our study is to bring more accuracy and precision to the study of topological indices, especially in the context of connectivity indices. FGs have less information in comparison with IFGs. In particular situations like vagueness and uncertainty, FGs are described by only membership grades, but IFGs are characterized by the two grades known by membership and nonmembership. Due to the description of opinions using two membership grades, IFGs have less information loss as compared to FGs. So, that is why we aim to propose the concepts of several $\mathscr{C} \mathscr{I} \mathcal{S}$ for IFGs and study their applications.

\section{Connectivity Index for Intuitionistic Fuzzy Graphs}

When we talk about the network like Internet or transport network, naturally, we think about the connectivity of this network. The connectivity means how stable and dynamic this network is! So, we can say that this measure of connectivity is the most fundamental and natural. The measure of connectivity is already available in FGs. But IFG is the generalization of FG, and it gives better results in situations where FGs are not preferable. So, because of this reason, we have proposed this concept of connectivity from FGs to IFGs. We have made some results of connectivity of FGs to IFGs. We define $\mathscr{C} \mathscr{I}$ formally as follows.

Definition 13. The $\mathscr{C} \mathscr{I}$ of an IFG $G=(N, M)$ is defined as

$$
\begin{aligned}
\mathscr{C} \mathscr{F}(G) & =\sum_{u, v \in V(G)}\left(T_{N}(u), F_{N}(u)\right)\left(T_{N}(v), F_{N}(v)\right) \times \operatorname{CONN}_{G}(u, v) \\
& =\sum_{u, v \in V(G)}\left(T_{N}(u), F_{N}(u)\right)\left(T_{N}(v), F_{N}(v)\right)\left(\operatorname{CONN}_{T(G)}(u, v), \operatorname{CONN}_{F(G)}(u, v)\right) \\
& =\sum_{u, v \in V(G)}\left(T_{N}(u) T_{N}(v) \operatorname{CONN}_{T(G)}(u, v)+F_{N}(u) F_{N}(v) \operatorname{CONN}_{F(G)}(u, v)\right) \\
& =\sum_{u, v \in V(G)} T_{N}(u) T_{N}(v) \operatorname{CONN}_{T(G)}(u, v)+\sum_{u, v \in V(G)} F_{N}(u) F_{N}(v) \operatorname{CONN}_{F(G)}(u, v) \\
& =\mathscr{T} \mathscr{C} \mathscr{I}(G)+\mathscr{F} \mathscr{C} \mathscr{I}(G),
\end{aligned}
$$

where $\operatorname{TCI}(G)$ is T-connectivity index of $G, \operatorname{FCI}(G)$ is F-connectivity index of $G$, and $\operatorname{CONN}_{T(G)}(u, v)$ and
$\operatorname{CONN}_{F(G)}(u, v)$ are T-strength of connectedness and F-strength of connectedness between $u$ and $v$ 
Example 4. Refer to Figure 1,

$$
\begin{aligned}
\mathscr{T} \mathscr{C} \mathscr{I}(G)= & \sum_{u, v \in V(G)} T_{N}(u) T_{N}(v) \operatorname{CONN}_{T(G)}(u, v) \\
= & (0.7)(0.6)(0.6) \\
& +(0.7)(0.5)(0.4)+(0.7)(0.4)(0.4)+(0.7)(0.6)(0.6) \\
& +(0.5)(0.4)(0.4)+(0.5)(0.6)(0.4)+(0.4)(0.6)(0.4) \\
= & 0.252+0.14+0.112+0.252+0.12+0.096+0.216+0.08+0.12+0.096 \\
= & 1.484, \\
& \sum_{\mathcal{F}} F_{N}(u) F_{N}(v) \operatorname{CONN}_{F(G)}(u, v) \\
= & (0.3)(0.3)(0.3)+(0.3)(0.4)(0.4)+(0.3)(0.6)(0.6)+(0.3)(0.4)(0.4) \\
& +(0.3)(0.4)(0.4)+(0.3)(0.6)(0.6)+(0.3)(0.4)(0.4)+(0.4)(0.6)(0.6) \\
& +(0.4)(0.4)(0.6)+(0.6)(0.4)(0.6) \\
= & 0.027+0.048+0.108+0.048+0.048+0.108+0.048+0.144+0.096+0.144 \\
= & 0.819 .
\end{aligned}
$$

$=2.303$.

So, $\mathscr{C} \mathscr{I}(G)=\mathscr{T} \mathscr{C} \mathscr{I}(G) \quad+\mathscr{F} \mathscr{C} \mathscr{I}(G)=1.484+0.819$

It may be observed that $\mathrm{TCI}(G)>\mathrm{FCI}(G)$, which shows that the level of FCI $(G)$ is lower than the level of TCI $(G)$ in this problem. This comparison is interesting and useful in applications of connectivity index.

Theorem 1. Let $G=(N, M)$ be a complete IFG with $N^{*}=$ $\left\{v_{1}, v_{2}, v_{3}, \ldots, v_{n}\right\}$ such that $t_{1} \leq t_{2} \leq \cdots \leq t_{n}$ and $s_{1} \geq s_{2} \geq s_{3} \geq \cdots \geq s_{n}$, where $t_{i}=T_{N}\left(v_{i}\right)$ and $s_{i}=F_{N}\left(v_{i}\right)$. Then,

$$
\mathscr{C} \mathscr{J}(G)=\sum_{i=1}^{n-1} t_{i}^{2} \sum_{j=i+1}^{n} t_{j}+\sum_{i=1}^{n-1} s_{i}^{2} \sum_{j=i+1}^{n} s_{j}
$$

Proof. Let $v_{1}$ be the vertex having least truth-membership value $t_{1}$. For a complete IFG, $\operatorname{CONN}_{T(G)}(u, v)=T_{M}(u, v)$ for each $u, v \in N^{*}$. So, $T_{M}\left(v_{1}, v_{i}\right)=t_{1} ; 2 \leq i \leq n$ and hence, $T_{N}\left(v_{1}\right) T_{N}\left(v_{i}\right) \operatorname{CONN}_{T(G)}\left(v_{1}, v_{i}\right)=t_{1} \cdot t_{i} \cdot t_{1}=t_{1}^{2} t_{i} ; 2 \leq i \leq n$. Taking summation over $i$, we have

$$
\sum_{i=2}^{n} T_{N}\left(v_{1}\right) T_{N}\left(v_{i}\right) \operatorname{CONN}_{T(G)}\left(v_{1}, v_{i}\right)=\sum_{i=2}^{n} t_{1}^{2} t_{i}
$$

Similarly, for vertex $v_{2}$, we obtain

$$
\sum_{i=3}^{n} T_{N}\left(v_{2}\right) T_{N}\left(v_{i}\right) \operatorname{CONN}_{T(G)}\left(v_{2}, v_{i}\right)=\sum_{i=3}^{n} t_{2}^{2} t_{i},
$$

and for vertex $v_{3}$

$$
\sum_{i=4}^{n} T_{N}\left(v_{3}\right) T_{N}\left(v_{i}\right) \operatorname{CONN}_{T(G)}\left(v_{3}, v_{i}\right)=\sum_{i=4}^{n} t_{3}^{2} t_{i},
$$

and so on, for vertex $v_{n-1}$

$$
\sum_{i=n}^{n} T_{N}\left(v_{n-1}\right) T_{N}\left(v_{i}\right) \operatorname{CONN}_{T(G)}\left(v_{n-1}, v_{i}\right)=\sum_{i=n}^{n} t_{n-1}^{2} t_{i} .
$$

By adding all the above equations, we get

$$
\begin{aligned}
\mathscr{T} \mathscr{C} \mathscr{I}(G) & =\sum_{i=2}^{n} t_{1}^{2} t_{i}+\sum_{i=3}^{n} t_{2}^{2} t_{i}+\sum_{i=4}^{n} t_{3}^{2} t_{i}+\cdots+\sum_{i=n}^{n} t_{n-1}^{2} t_{i} \\
& =\sum_{i=1}^{n-1} t_{i}^{2} \sum_{j=i+1}^{n} t_{j} .
\end{aligned}
$$

Now, let $v_{1}$ be the vertex with the largest falsity-membership value $s_{1}$. For a complete IFG, $\operatorname{CONN}_{F(G)}(u, v)=$ $F_{M}(u, v)$ for each $u, v \in N^{*}$. Thus, $F_{M}\left(v_{1}, v_{i}\right)=s_{1} ; 2 \leq i \leq n$ and

hence,

$F_{N}\left(v_{1}\right) F_{N}\left(v_{i}\right) \operatorname{CONN}_{F(G)}\left(v_{1}, v_{i}\right)=s_{1} \cdot s_{i} \cdot s_{1}=s_{1}^{2} s_{i} ; 2 \leq i \leq n$. Summing over $i$, we have

$$
\sum_{i=2}^{n} F_{N}\left(v_{1}\right) F_{N}\left(v_{i}\right) \operatorname{CONN}_{F(G)}\left(v_{1}, v_{i}\right)=\sum_{i=2}^{n} s_{1}^{2} s_{i} .
$$

Similarly, for vertex $v_{2}$, we have

$$
\sum_{i=3}^{n} F_{N}\left(v_{2}\right) F_{N}\left(v_{i}\right) \operatorname{CONN}_{F(G)}\left(v_{2}, v_{i}\right)=\sum_{i=3}^{n} s_{2}^{2} s_{i},
$$

and for vertex $v_{3}$

$$
\sum_{i=4}^{n} F_{N}\left(v_{3}\right) F_{N}\left(v_{i}\right) \operatorname{CONN}_{F(G)}\left(v_{3}, v_{i}\right)=\sum_{i=4}^{n} s_{3}^{2} s_{i},
$$

and so on, for vertex $v_{n-1}$ 
$\sum_{i=n}^{n} F_{N}\left(v_{n-1}\right) F_{N}\left(v_{i}\right) \operatorname{CONN}_{F(G)}\left(v_{n-1}, v_{i}\right)=\sum_{i=n}^{n} s_{n-1}^{2} s_{i}$.

By adding all the above equations, we get

$$
\begin{aligned}
\mathscr{F} \mathscr{C} \mathscr{I}(G)= & \sum_{i=2}^{n} s_{1}^{2} s_{i}+\sum_{i=3}^{n} s_{2}^{2} s_{i}+\sum_{i=4}^{n} s_{3}^{2} s_{i} \\
& +\cdots+\sum_{i=n}^{n} s_{n-1}^{2} s_{i}=\sum_{i=1}^{n-1} s_{i}^{2} \sum_{j=i+1}^{n} s_{j} .
\end{aligned}
$$

Hence, by the definition of connectivity, we see

$$
\begin{aligned}
\mathscr{C} \mathscr{I}(G) & =\mathscr{T} \mathscr{C} \mathscr{I}(G)+\mathscr{F} \mathscr{C} \mathscr{I}(G) \\
& =\sum_{i=1}^{n-1} t_{i}^{2} \sum_{j=i+1}^{n} t_{j}+\sum_{i=1}^{n-1} s_{i}^{2} \sum_{j=i+1}^{n} s_{j} .
\end{aligned}
$$

Example 5. In Figure 4, it can be easily seen that $k_{3}$ is a complete IFG. So,

$$
\begin{aligned}
\mathscr{T} \mathscr{C} \mathscr{I}(G) & =\sum_{i=1}^{3} T_{N}\left(v_{i}\right) T_{N}\left(v_{j}\right) \operatorname{CONN}_{T(G)}\left(v_{i}, v_{j}\right) \\
& =(0.4)(0.5)(0.4)+(0.5)(0.5)(0.5)+(0.4)(0.5)(0.4) \\
& =0.285, \\
\mathscr{F} \mathscr{C} \mathscr{S}(G) & =\sum_{i=1}^{3} F_{N}\left(v_{i}\right) F_{N}\left(v_{j}\right) \operatorname{CONN}_{F(G)}\left(v_{i}, v_{j}\right) \\
& =(0.5)(0.4)(0.5)+(0.4)(0.3)(0.4)+(0.5)(0.3)(0.5) \\
& =0.223 .
\end{aligned}
$$

Therefore, $\mathscr{C} \mathscr{I}(G)=\mathscr{T} \mathscr{C} \mathscr{I}(G)+\mathscr{F} \mathscr{C} \mathscr{I}(G)=0.285+0.223=0.508$.
Now, we use above theorem

$\sum_{i=1}^{n-1} t_{i}^{2} \sum_{j=i+1}^{n} t_{j}=\sum_{i=1}^{2} t_{i}^{2} \sum_{j=i+1}^{3} t_{j}=t_{1}^{2}\left(t_{2}+t_{3}\right)+t_{2}^{2} t_{3}$

$=(0.4)^{2}(0.5+0.5)+(0.5)^{2}(0.5)=0.16+0.125=0.285$,

$\sum_{i=1}^{n-1} s_{i}^{2} \sum_{j=i+1}^{n} s_{j}=\sum_{i=1}^{2} s_{i}^{2} \sum_{j=i+1}^{3} s_{j}=s_{1}^{2}\left(s_{2}+s_{3}\right)+s_{2}^{2} s_{3}$

$=(0.5)^{2}(0.4+0.3)+(0.4)^{2}(0.3)=0.175+0.048=0.223$.

Adding these two summations, we get

$$
\sum_{i=1}^{n-1} t_{i}^{2} \sum_{j=i+1}^{n} t_{j}+\sum_{i=1}^{n-1} s_{i}^{2} \sum_{j=i+1}^{n} s_{j}=0.285+0.223=0.508 \text {. }
$$

Hence, it is verified that

$$
\mathscr{C} \mathscr{J}(G)=\sum_{i=1}^{n-1} t_{i}^{2} \sum_{j=i+1}^{n} t_{j}+\sum_{i=1}^{n-1} s_{i}^{2} \sum_{j=i+1}^{n} s_{j}
$$

\section{Edge Deleted and Vertex Deleted IFGs with Connectivity Index}

The $\mathscr{C} \mathscr{I}$ is affected or not by deleting a vertex or an edge. It is based on the nature of vertex and edge to be removed.

Example 6. Taking the $\mathscr{C} \mathscr{I}=2.017 G=(N, M)$ shown in Figure 5. Here, $\left(v_{1}, v_{4}\right),\left(v_{2}, v_{3}\right),\left(v_{4}, v_{5}\right)$ are $\alpha$-strong arcs, $\left(v_{1}, v_{3}\right),\left(v_{3}, v_{4}\right)$ are $\beta$-strong arcs, and $\left(v_{1}, v_{2}\right),\left(v_{3}, v_{5}\right)$ are $\delta$-arcs. Then,

$$
\begin{aligned}
\mathscr{T} \mathscr{C} \mathscr{I}(G)= & \sum_{i=1}^{10} T_{N}\left(v_{i}\right) T_{N}\left(v_{j}\right) \operatorname{CONN}_{T(G)}\left(v_{i}, v_{j}\right) \\
= & (0.5)(0.3)(0.3)+(0.5)(0.4)(0.3)+(0.5)(0.6)(0.4)+(0.5)(0.1)(0.1) \\
& +(0.3)(0.4)(0.3)+(0.3)(0.6)(0.3)+(0.3)(0.1)(0.1)+(0.4)(0.6)(0.3) \\
& +(0.4)(0.1)(0.1)+(0.6)(0.1)(0.1)=0.405, \\
\mathscr{F} \mathscr{C} \mathscr{I}(G)= & \sum_{i=1}^{10} F_{N}\left(v_{i}\right) F_{N}\left(v_{j}\right) \operatorname{CONN}_{F(G)}\left(v_{i}, v_{j}\right) \\
= & (0.4)(0.5)(0.5)+(0.4)(0.4)(0.5)+(0.4)(0.4)(0.4)+(0.4)(0.8)(0.8) \\
& +(0.5)(0.4)(0.5)+(0.5)(0.4)(0.5)+(0.5)(0.8)(0.8)+(0.4)(0.4)(0.5) \\
& +(0.4)(0.8)(0.8)+(0.4)(0.8)(0.8)=1.612 .
\end{aligned}
$$

So, we have $\mathscr{C} \mathscr{I}(G)=\mathscr{T} \mathscr{C} \mathscr{I}(G)+\mathscr{F} \mathscr{C} \mathscr{I} \quad(G)=$ $0.405+1.612=2.017$.

So, we have $\mathscr{C} \mathscr{I}\left(G-\left(v_{1}, v_{4}\right)\right)=\mathscr{T} \mathscr{C} \mathscr{I}\left(G-\left(v_{1}, v_{4}\right)\right)$ $+\mathscr{F} \mathscr{C} \mathscr{I}\left(G-\left(v_{1}, v_{4}\right)\right)=0.375+1.628=2.003$. Thus, $\mathscr{C} \mathscr{I}$ $\left(G-\left(v_{1}, v_{4}\right)\right)<\mathscr{C} \mathscr{I}(G)$, which means that $\mathscr{C} \mathscr{I}$ of $G$ has been reduced by deleting $\alpha$-strong edge $\left(v_{1}, v_{4}\right)$. The IFG,
$G-\left(v_{1}, v_{2}\right)$ is shown in Figure 6(a). If we delete the $\beta$-strong edge $\left(v_{1}, v_{3}\right)$, then the strength of connectedness between every pair of vertices is invariant, i.e., $\operatorname{CONN}_{T(G)}\left(v_{i}, v_{j}\right)=$ $\mathrm{CONN}_{T(G)-\left(V_{1}, V_{3}\right)}\left(v_{i}, v_{j}\right)$ and $\operatorname{CONN}_{F(G)}\left(v_{i}, v_{j}\right)=$ $\operatorname{CONN}_{F(G)-\left(V_{1}, V_{3}\right)}\left(v_{i}, v_{j}\right)$, so $\mathscr{C} \mathscr{I}\left(G-\left(v_{1}, v_{3}\right)\right)=\mathscr{C} \mathscr{I}(G)$. The graph of $G-\left(v_{1}, v_{3}\right)$ is shown in Figure 6(b). Similarly, 


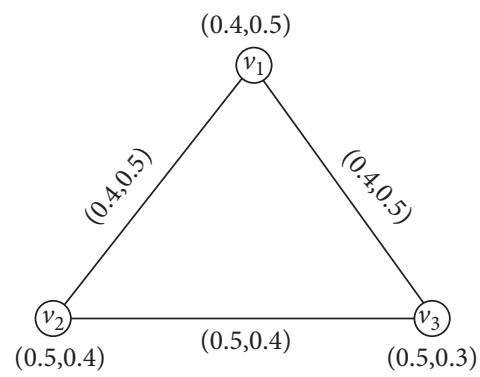

FIgURe 4: A complete IFG with $\mathscr{C} \mathscr{I}=0.508$.

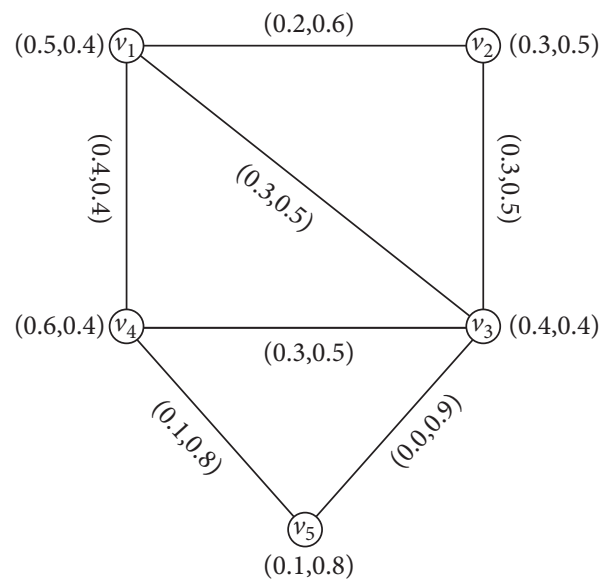

Figure 5: An IFG with $\mathscr{C} \mathscr{I}=2.017$.

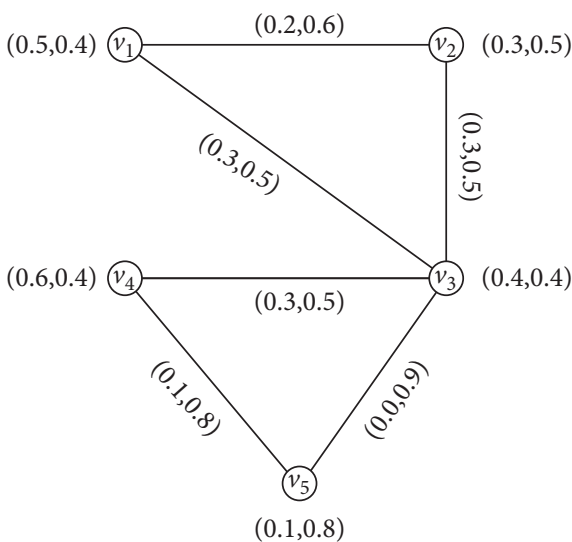

(a)

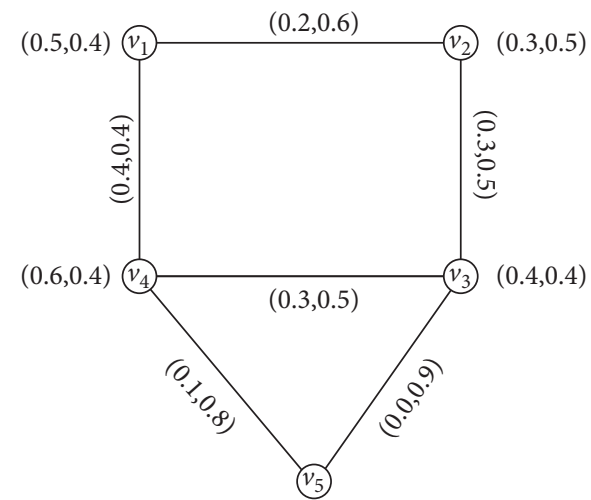

$(0.1,0.8)$

(b)

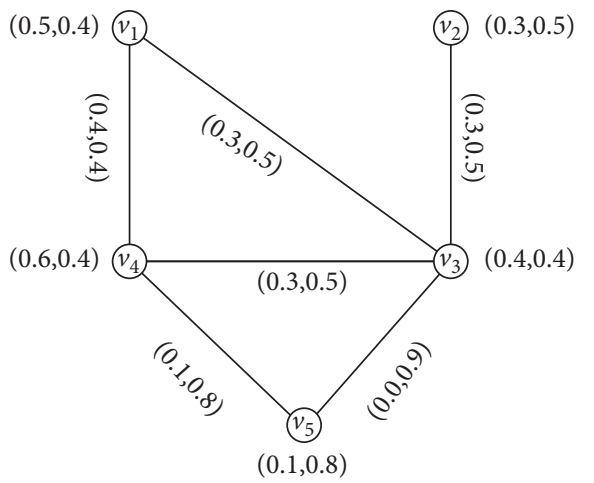

(c)

Figure 6: $G-\left(v_{1}, v_{4}\right), G-\left(v_{1}, v_{3}\right)$ and $G-\left(v_{1}, v_{2}\right)$. (a) $G-\left(v_{1}, v_{4}\right)$. (b) $G-\left(v_{1}, v_{3}\right)$. (c) $G-\left(v_{1}, v_{2}\right)$. 
when we delete the $\delta$-arc $\left(v_{1}, v_{2}\right)$, then the strength of connectedness between every pair of vertices does not change, and so is the $\mathscr{C} \mathscr{I}$. The graph of $G-\left(v_{1}, v_{2}\right)$ is shown in Figure 6(c).

$$
\begin{aligned}
\mathscr{T} \mathscr{C} \mathscr{I}\left(G-\left(v_{1}, v_{4}\right)\right)= & \sum_{i=1}^{10} T_{N}\left(v_{i}\right) T_{N}\left(v_{j}\right) \operatorname{CONN}_{T(G)-\left(v_{1}, v_{4}\right)}\left(v_{i}, v_{j}\right) \\
= & (0.5)(0.3)(0.3)+(0.5)(0.4)(0.3)+(0.5)(0.6)(0.3) \\
& +(0.5)(0.1)(0.1)+(0.3)(0.4)(0.3)+(0.3)(0.6)(0.3) \\
& +(0.3)(0.1)(0.1)+(0.4)(0.6)(0.3)+(0.4)(0.1)(0.1) \\
& +(0.6)(0.1)(0.1)=0.375, \\
\mathscr{F} \mathscr{C} \mathscr{I}\left(G-\left(v_{1}, v_{4}\right)\right)= & \sum_{i=1}^{10} F_{N}\left(v_{i}\right) F_{N}\left(v_{j}\right) \operatorname{CONN}_{F(G)-\left(v_{1}, v_{4}\right)}\left(v_{i}, v_{j}\right) \\
= & (0.4)(0.5)(0.5)+(0.4)(0.4)(0.5)+(0.4)(0.4)(0.5) \\
& +(0.4)(0.8)(0.8)+(0.5)(0.4)(0.5)+(0.5)(0.4)(0.5) \\
& +(0.5)(0.8)(0.8)+(0.4)(0.4)(0.5)+(0.4)(0.8)(0.8) \\
& +(0.4)(0.8)(0.8)=1.628 .
\end{aligned}
$$

Theorem 2. Let $H$ be the IF subgraph of an IFG $G=(N, M)$ formed by removing an edge $u v \in M_{G}$ from $G$. Then, $\mathscr{C} \mathscr{I}(G)>\mathscr{C} \mathscr{I}(H)$ or $\mathscr{C} \mathscr{I}(G)<\mathscr{C} \mathscr{I}(H)$ iff $u v$ is a bridge.

Proof. Take $u v$ as a bridge. According to the definition, there exit $u$ and $v$ such that their strength of connectedness will be decreased. So, we conclude that $\mathscr{C} \mathscr{I}(G)>\mathscr{C} \mathscr{I}(H)$ or $\mathscr{C} \mathscr{I}(G)<\mathscr{C} \mathscr{I}(H)$.

Conversely, suppose that $\mathscr{C} \mathscr{I}(G)>\mathscr{C} \mathscr{I}(H)$ or $\mathscr{C} \mathscr{I}(G)<\mathscr{C} \mathscr{I}(H)$ and consider the possibilities given below.

Case 1: suppose that $u v$ is a $\delta$-arc. Then, $\mathrm{CONN}_{T(G)-u v}(u, v)=\mathrm{CONN}_{T(G)}(u, v) \quad$ and $\mathrm{CONN}_{F(G)-u v}(u, v)=\mathrm{CONN}_{F(G)}(u, v)$. So, we have $\mathscr{T} \mathscr{C} \mathscr{I}(G)=\mathscr{T} \mathscr{C} \mathscr{I}(H)$ and $\mathscr{F} \mathscr{C} \mathscr{I}(G)=\mathscr{F} \mathscr{C} \mathscr{I}(H)$ and therefore, $\mathscr{C} \mathscr{I}(G)=\mathscr{C} \mathscr{I}(H)$.

Case 2: take $u v$ as $\beta$-strong edge. Then, $T_{M}(u, v)=$ $\operatorname{CONN}_{T(G)-u v}(u, v), F_{M}(u, v)=\operatorname{CONN}_{F(G)-u v}(u, v)$. This implies that there is another $u-v$ path different from $u v$ edge. Therefore, the removal of the arc $u v$ will have no effect on the strength of connectedness between $u$ and $v$. So, $\mathscr{C} \mathscr{I}(G)=\mathscr{C} \mathscr{I}(H)$.

Case 3: now, take $u v$ as $\alpha$-strong edge. Then, $T_{M}(u, v)>\mathrm{CONN}_{T(G)-u v}(u, v), \quad F_{M}(u, v)<\mathrm{CONN}$ $F(G)-u v(u, v)$. So, the only strongest path is $u v$ edge having strength equal to $\left(T_{M}(u, v), F_{M}(u, v)\right)$. Then, clearly $\mathscr{C} \mathscr{I}(G)>\mathscr{C} \mathscr{I}(H)$, or $\mathscr{C} \mathscr{I}(G)<\mathscr{C} \mathscr{I}(H)$ $\mathscr{T} \mathscr{C} \mathscr{I}(G)-\mathscr{T} \mathscr{C} \mathscr{I}(H)>\mathscr{F} \mathscr{C} \mathscr{I}(G)-\mathscr{F} \mathscr{C} \mathscr{I}(H)$ or $\mathscr{T} \mathscr{C} \mathscr{I}(G)-\mathscr{T} \mathscr{C} \mathscr{I}(H)<\quad \mathscr{F} \mathscr{C} \mathscr{I}(G)-\mathscr{F} \mathscr{C} \mathscr{I}(H)$ since $\alpha$-strong edges are IF bridges. This implies that $u v$ is a bridge.
Corollary 1. Let $H$ be the IF subgraph of an IFG $G=(N, M)$ formed by removing an edge $u v \in M_{G}$ from $G$. Then, $\mathscr{C} \mathscr{I}(G)=\mathscr{C} \mathscr{I}(H)$ iff $u v$ is either $\delta$ edge or $\beta$-strong.

Corollary 2. Suppose that uv an edge of a complete IFG $G=(N, M)$. Then, $\mathscr{C} \mathscr{I}(G) \neq \mathscr{C} \mathscr{I}(G-u v)$ iff $u v$ is a unique IF bridge of $G$.

Proof. Let $G=(N, M)$ be a complete IFG. Suppose that $\mathscr{C} \mathscr{I}(G) \neq \mathscr{C} \mathscr{I}(G-u v)$. Then, $u v$ is the only IF bridge of $G$.

Conversely, let $u v$ be a unique IF bridge of $G$. Hence, by Theorem 2, it follows that $\mathscr{C} \mathscr{I}(G) \neq \mathscr{C} \mathscr{I}(G-u v)$.

Theorem 3. Let $G_{1}=\left(N_{1}, M_{1}\right)$ and $G_{2}=\left(N_{1}, M_{2}\right)$ be the two isomorphic IFGs. Then, $\mathscr{C} \mathscr{I}\left(G_{1}\right)=\mathscr{C} \mathscr{I}\left(G_{2}\right)$.

Proof. Suppose that $G_{1}=\left(N_{1}, M_{1}\right)$ and $G_{2}=\left(N_{2}, M_{2}\right)$ are isomorphic IFGs. Then, $\exists$ is a mapping $h: N_{1} \longrightarrow N_{2}$ such that $h$ is bijective and $T_{N_{1}}\left(u_{i}\right)=T_{N_{2}}\left(h\left(u_{i}\right)\right)$ and $F_{N_{1}}\left(u_{i}\right)=F_{N_{2}}\left(h\left(u_{i}\right)\right)$ for all $u_{i} \in N^{*}$ as well as $T_{M_{1}}\left(u_{i}, u_{j}\right)=T_{M_{2}}\left(h\left(u_{i}\right), h\left(u_{j}\right)\right)$ and $F_{M_{1}}\left(u_{i}, u_{j}\right)=$ $F_{M_{2}}\left(h\left(u_{i}\right), h\left(u_{j}\right)\right)$ for $\left(u_{i}, u_{j}\right) \in M^{*}$. As $G_{1}$ and $G_{2}$ are isomorphic, then the strength of any strongest path between $u_{i}$ and $u_{j}$ is equal to that between $h\left(u_{i}\right)$ and $h\left(u_{j}\right)$ in $G_{2}$. Thus, for $u, v \in N^{*}$

$$
\begin{aligned}
\operatorname{CONN}_{T\left(G_{1}\right)}\left(u_{i}, u_{j}\right) & =\operatorname{CONN}_{T\left(G_{2}\right)}\left(h\left(u_{i}\right), h\left(u_{j}\right)\right), \\
\mathrm{CONN}_{F\left(G_{1}\right)}\left(u_{i}, v u_{j}\right) & =\mathrm{CONN}_{F\left(G_{2}\right)}\left(h\left(u_{i}\right), h\left(u_{j}\right)\right) .
\end{aligned}
$$

So, we have 


$$
\begin{aligned}
\mathscr{T} \mathscr{C} \mathscr{F}\left(G_{1}\right)= & \sum_{u_{i}, u_{j} \in V\left(G_{1}\right)} T_{N_{1}}\left(u_{i}\right) T_{N_{1}}\left(u_{j}\right) \operatorname{CONN}_{T\left(G_{1}\right)}\left(u_{i}, u_{j}\right) \\
= & \sum_{h\left(u_{i}\right), h\left(u_{j}\right) \in V\left(G_{2}\right)} T_{N_{2}}\left(h\left(u_{i}\right)\right) T_{N_{2}}\left(h\left(u_{j}\right)\right) \operatorname{CONN}_{T\left(G_{2}\right)}\left(h\left(u_{i}\right), h\left(u_{j}\right)\right) \\
= & \mathscr{T} \mathscr{C} \mathscr{F}\left(G_{2}\right), \\
\mathscr{F} \mathscr{C} \mathscr{I}\left(G_{1}\right)= & \sum_{u_{i}, u_{j} \in V\left(G_{1}\right)} F_{N_{1}}\left(u_{i}\right) F_{N_{1}}\left(u_{j}\right) \operatorname{CONN}_{F\left(G_{1}\right)}\left(u_{i}, u_{j}\right) \\
= & \sum_{h\left(u_{i}\right), h\left(u_{j}\right) \in V\left(G_{2}\right)} F_{N_{2}}\left(h\left(u_{i}\right)\right) F_{N_{2}}\left(h\left(u_{j}\right)\right) \operatorname{CONN}_{F}\left(G_{2}\right) \\
= & \mathscr{F} \mathscr{C} \mathscr{I}\left(G_{2}\right) .
\end{aligned}
$$

Thus, $\mathscr{T} \mathscr{C} \mathscr{I}\left(G_{1}\right)+\mathscr{F} \mathscr{C} \mathscr{I}\left(G_{2}\right)=\mathscr{T} \mathscr{C} \mathscr{F}\left(G_{2}\right)+\mathscr{F} \mathscr{C} \mathscr{I}\left(G_{2}\right)$. This implies that $\mathscr{C} \mathscr{I}\left(G_{1}\right)=\mathscr{C} \mathscr{I}\left(G_{2}\right)$.

\section{Strongest Strong Cycles, $\boldsymbol{\theta}$-Evaluation of Vertices, Cycle Connectivity, and CI of Strong Cycle}

This section contains some concepts about IF cycles. IF strongest strong cycles, $\theta$-evaluation of IF vertices, IF cycle connectivity, and $\mathscr{C} \mathscr{I}$ of IF strong cycles are defined in the current section. Also, some properties related to these concepts are studied.

Definition 14. The truth and falsity values of the weakest edge in a cycle $C$ are defined to be the strength of $C$ in an IFG G.

Definition 15. Let $C$ denote a cycle in an IFG $G$. Then, $C$ is called IF strongest strong cycle (IFSSC) if it is the union of two strongest strong $u-v$ paths for each of $u$ and $v$ in $C$ with the exception when $u v$ in $C$ is an IF bridge of $G$.

Remark 1. We observe that when $u v$ is an IF bridge of $G$ and it lies in $C$, the condition for $C$ to be the union of two strongest strong $u$ to $v$ paths can be omitted for $u$ and $v$. Also, $\operatorname{CONN}_{T(G)}(a, b)=\operatorname{CONN}_{T(C)}(a, b)$ and $\operatorname{CONN}_{F(G)}(a, b)=$ $\operatorname{CONN}_{F(C)}(a, b) \forall a, b \in G$.

Definition 16. If $C$ is a cycle in an IFG $G$, then $C$ is said to be strong, provided each of its edges is strong.

Example 7. In Figure 7, we take $\left(T_{N}(u), F_{N}(u)\right)=(0.6,0.3)$ for all $u \in N^{*}$. The edges $\left(v_{1}, v_{2}\right),\left(v_{3}, v_{5}\right)$ are bridges in $G$. $C_{1}=v_{1} v_{2} v_{3} v_{5} v_{1}$ and $C_{2}=v_{5} v_{3} v_{4} v_{5}$ are strongest strong cycles as $C_{3}=v_{1} v_{2} v_{3} v_{4} v_{5} v_{1}$ is not the union of two strongest strong $v_{3}-v_{5}$ paths. So, it is not a strong cycle. Also, $\operatorname{CONN}_{T(G)}\left(v_{3}, v_{5}\right)=0.6$ and $\operatorname{CONN}_{F(G)}\left(v_{3}, v_{5}\right)=0.3$. But $C_{3}$ has no strongest $v_{3}-v_{5}$ path. Moreover, we see that $\left(v_{3}, v_{5}\right)$ is a fuzzy bridge of $G$ that is outside of $C_{3}$.
Definition 17. Let $G$ be an IFG. Then, $T \theta$-evaluation of two vertices $u_{i}$ and $u_{j}$ in $G$ is the set $\theta_{T}\left(u_{i}, u_{j}\right)$ defined by

$$
\theta_{T}\left(u_{i}, u_{j}\right)=\{\alpha: \alpha \in(0,1]\},
$$

where $\alpha$ represents $T$-strength of a SC passing through both $u_{i}$ and $u_{j}$. Similarly, $F \theta$-evaluation of $u_{i}$ and $u_{j}$ is the set $\theta_{F}\left(u_{i}, u_{j}\right)$ defined by

$$
\theta_{F}(u, v)=\{\beta: \beta \in(0,1]\},
$$

where $\beta$ represents $F$-strength of the same SC passing through both $u_{i}$ and $u_{j}$.

Note 1. If cycles through $u$ and $v$ do not exist, then $\theta_{T}\left(u_{i}, u_{j}\right)=\phi$ and $\theta_{F}\left(u_{i}, v_{j}\right)=\phi$. With this $\theta$-evaluation, we define another connectivity measure in IFGs called cyclic connectivity (CC).

Definition 18. Let $G$ be an IFG. Then, cycle T-connectivity between $u_{i}$ and $u_{j}$ in $G$ is denoted and defined by

$$
\mathscr{T} \mathscr{C}_{u_{i}, u_{j}}^{G}=\vee\left\{\alpha: \alpha \in \theta_{T}\left(u_{i}, u_{j}\right) ; u_{i}, u_{j} \in N^{*}\right\} .
$$

Similarly, cycle F-connectivity between $u_{i}$ and $u_{j}$ in $G$ is denoted and defined by

$$
\mathscr{F} \mathscr{C}_{u_{i}, u_{j}}^{G}=\wedge\left\{\beta: \beta \in \theta_{F}\left(u_{i}, u_{j}\right) ; u_{i}, u_{j} \in N^{*}\right\} .
$$

Note 2. If $\theta_{T}\left(u_{i}, u_{j}\right)=\phi$ and $\theta_{F}\left(u_{i}, u_{j}\right)=\phi$ for any two vertices $u_{i}$ and $u_{j}$, then we define cycle T-connectivity and cycle F-connectivity to be zero, i.e., $\mathscr{T}_{u}^{G}{ }_{u, v}^{G}=0$ and $\mathscr{F} \mathscr{C}_{u, v}^{G}=0$.

Example 8. From Figure 8, we have $\theta_{T}\left(v_{1}, v_{3}\right)=\{0.7,0.6\}$ and hence, $\mathscr{T}_{\mathscr{C}_{v_{1}, v_{3}}^{G}}^{G}=0.7$. Similarly, $\theta_{F}\left(v_{1}, v_{3}\right)=\{0.3,0.4\}$ and therefore, $\mathscr{F}_{\mathscr{C}_{1}, v_{3}}^{G}=0.3$.

Theorem 4. Let $G$ be an IFG and for any $u_{i}, u_{j} \in N^{*}$, both $u_{i}$ and $u_{j}$ lie on a common SC. Then, 


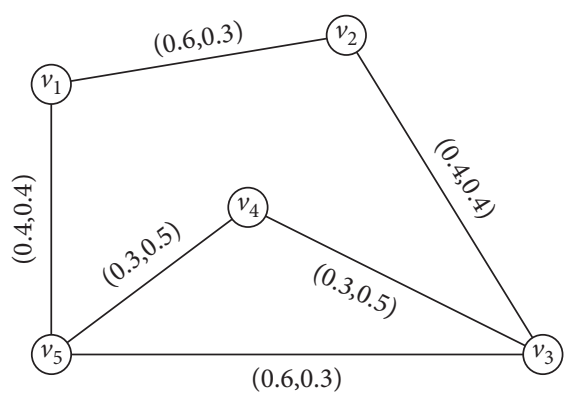

FIgURE 7: Strongest strong cycles.

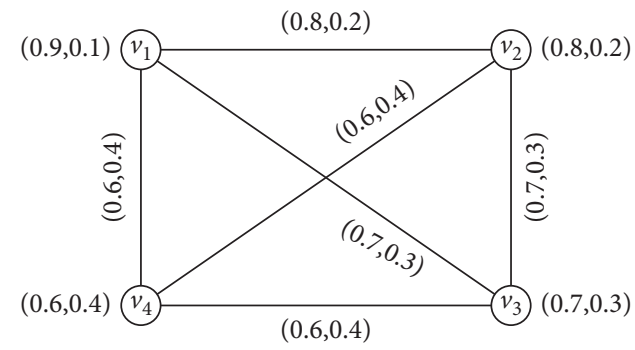

Figure 8: The cycle connectivity.

$$
\mathscr{C} \mathscr{F}(G)=\sum_{u_{i}, u_{j} \in N^{*}} T_{N}\left(u_{i}\right) T_{N}\left(u_{j}\right) \mathscr{T} \mathscr{C}_{u_{i}, u_{j}}^{G}+\sum_{u_{i}, u_{j} \in N^{*}} F_{N}\left(u_{i}\right) F_{N}\left(u_{j}\right) \mathscr{F} \mathscr{C}_{u_{i}, u_{j}}^{G}
$$

Proof. Suppose that $u_{i}, u_{j} \in N^{*}$ such that both of them lie on a common IFSSC. Then, $\mathscr{T} \mathscr{C}_{u_{i}, u_{j}}^{G}=\max \{\alpha$ : $\left.\alpha \in \theta_{T}\left(u_{i}, u_{j}\right) ; u_{i}, u_{j} \in N^{*}\right\}$, where $\theta_{T}\left(u_{i}, u_{j}\right)=\{\alpha \in(0,1$ ]: $\alpha$ is $T$ - strength of a strong cycle through $u$ and $v\}$. Therefore, $\operatorname{CONN}_{T(G)}\left(u_{i}, u_{j}\right)=\mathscr{T} \mathscr{C}_{u_{i}, u_{j}}^{G}$ and hence,

$$
\mathscr{T} \mathscr{C} \mathscr{F}(G)=\sum_{u_{i}, u_{j} \in N^{*}} T_{N}\left(u_{i}\right) T_{N}\left(u_{j}\right) \mathscr{T} \mathscr{C}_{u_{i}, u_{j}}^{G}
$$

Similarly, $\mathscr{F} \mathscr{C}_{u_{i}, u_{i}}^{G}=\min \left\{\beta: \beta \in \theta_{F}\left(u_{i}, u_{j}\right) ; u_{i}, u_{j} \in N^{*}\right\}$, where $\theta_{F}\left(u_{i}, u_{j}\right)=\{\beta \in(0,1]: \beta$ is $F-$ strength of a strong cycle through cyclethrough $u$ and $v$ \}. Thus, we obtain $\operatorname{CONN}_{F(G)}\left(u_{i}, u_{j}\right)=\mathscr{F} \mathscr{C}_{u_{i}, u_{j}}^{G}$ and hence,

$$
\mathscr{F} \mathscr{C} \mathscr{I}(G)=\sum_{u_{i}, u_{j} \in N^{*}} F_{N}\left(u_{i}\right) F_{N}\left(u_{j}\right) \mathscr{F} \mathscr{C}_{u_{i}, u_{j}}^{G} \text {. }
$$

So, we obtain

$$
\mathscr{C} \mathscr{F}(G)=\sum_{u_{i}, u_{j} \in N^{*}} T_{N}\left(u_{i}\right) T_{N}\left(u_{j}\right) \mathscr{T} \mathscr{C}_{u_{i}, u_{j}}^{G}+\sum_{u_{i}, u_{j} \in N^{*}} F_{N}\left(u_{i}\right) F_{N}\left(u_{j}\right) \mathscr{F} \mathscr{C}_{u_{i}, u_{j}}^{G}
$$

\section{Average Connectivity Index of an IFG}

The concept of average connectivity index is present in the literature of FGs. So, we introduced this concept for IFGs. The stability of a network is guaranteed by its average flow.

Example 9. Let $G=(N, M)$ be the IFG in Figure 9, with $\left(T_{N}(v), F_{N}(v)\right)=(0.9,0.1)$ for all $v \in N^{*}$. Then, by routine calculations, we have $\mathscr{T} \mathscr{C} \mathscr{F}(G)=1.944, \mathscr{F} \mathscr{C} \mathscr{F}(G)=0.006$ and $\mathscr{C} \mathscr{I}(G)=1.95$.

From Example 10, $\mathscr{C} \mathscr{F}(G)=1.95$ and the number of pairs in $G$ is $\left(\begin{array}{l}4 \\ 2\end{array}\right)=6$. By averaging the
$\mathscr{T} \mathscr{C} \mathscr{I}(G), \mathscr{F} \mathscr{C} \mathscr{I}(G) \quad$ and $\mathscr{C} \mathscr{I}(G)$, we get $\mathscr{A} \mathscr{T} \mathscr{C} \mathscr{I}(G)=(1 / 6) \mathscr{T} \mathscr{C} \mathscr{I}(G)=(1 / 6)(1.944)=0.324$, $\mathscr{A} \mathscr{F} \mathscr{C} \mathscr{I}(G)=(1 / 6) \mathscr{F} \mathscr{C} \mathscr{J}(G)=(1 / 6)(0.006)=0.001$ and $\mathscr{A} \mathscr{C} \mathscr{I}(G)=0.324+0.001=0.325$. Now, consider $G-V_{4}$, and we have $\mathscr{T} \mathscr{C} \mathscr{I}\left(G-v_{4}\right)=1.701, \mathscr{F} \mathscr{C} \mathscr{I}\left(G-v_{4}\right)=0.003$ and $\mathscr{C} \mathscr{I}\left(G-v_{4}\right)=1.701+0.003=1.704$. On averaging them, we have $\mathscr{A} \mathscr{T} \mathscr{C} \mathscr{I}\left(G-v_{4}\right)=(1.701 \quad / 3)=$ $0.567, \mathscr{A} \mathscr{F} \mathscr{C} \mathscr{I}\left(G-v_{4}\right)=(0.003 / 3)=0.001$ and $\mathscr{A} \mathscr{C} \mathscr{J}$ $\left(G-v_{4}\right)=0.567+0.001=0.568$. The overall connectivity of $G$ is increased by deleting vertex $v_{4}$ from $G$. The following definitions and results are led by this example.

Definition 19. Let $G=(N, M)$ be an IFG. Then, the average $T$-connectivity index of $G$ is denoted and defined as 


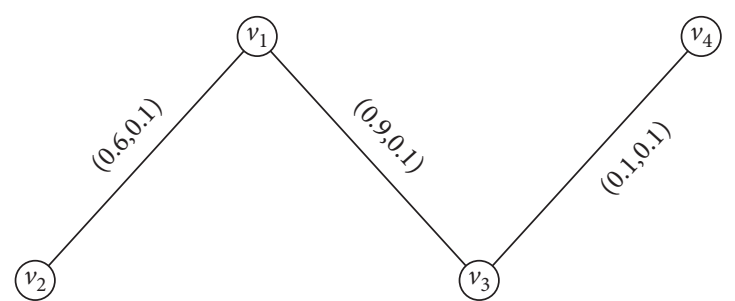

(a)

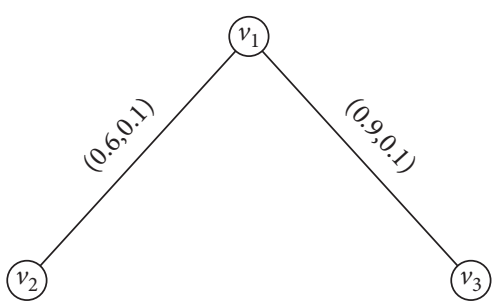

(b)

Figure 9: An IFG $G$ and $G-v_{4}$. (a) An IFG with $\mathscr{C} \mathscr{I}(G)=1.95$. (b) $G-v_{4}$.

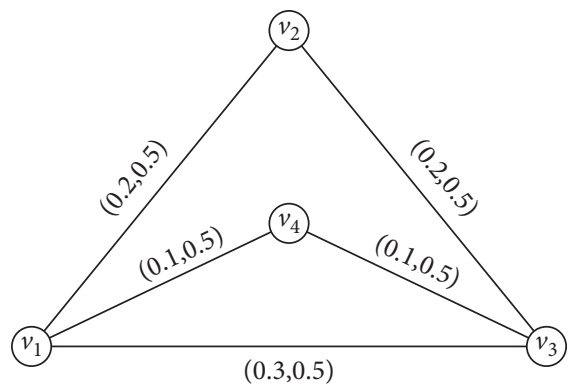

FIGURe 10: IFG with IFCRN, IFCEN, and IF neutral nodes.

$$
\mathscr{A} \mathscr{T} \mathscr{C} \mathscr{F}(G)=\frac{1}{\left(\begin{array}{l}
n \\
2
\end{array}\right)} \sum_{u, v \in N^{*}} T_{N}(u) T_{N}(v) \operatorname{CONN}_{T(G)}(u, v)
$$

and the average $F$-connectivity index of $G$ is denoted and defined as

$$
\mathscr{A} \mathscr{F} \mathscr{C} \mathscr{I}(G)=\frac{1}{\left(\begin{array}{c}
n \\
2
\end{array}\right)} \sum_{u, v \in N^{*}} F_{N}(u) F_{N}(v) \operatorname{CONN}_{F(G)}(u, v),
$$

where $\mathrm{CONN}_{T(G)}(u, v)$ is the $T$ - strength of connectedness, and $\mathrm{CONN}_{F(G)}(u, v)$ is the $F$ - strength of connectedness between the nodes $u$ and $v$.

Definition 20. Let $G=(N, M)$ be an IFG. The average connectivity index of $G$ is defined to be the sum of average $T$ connectivity index and average $F$ - connectivity index of $G$, i.e.,

$$
\begin{aligned}
\mathscr{A} \mathscr{C} \mathscr{I}(G)= & \frac{1}{\left(\begin{array}{c}
n \\
2
\end{array}\right)} \sum_{u, v \in N^{*}} T_{N}(u) T_{N}(v) \operatorname{CONN}_{T(G)}(u, v) \\
& +\frac{1}{\left(\begin{array}{l}
n \\
2
\end{array}\right)} \sum_{u, v \in N^{*}} F_{N}(u) F_{N}(v) \operatorname{CONN}_{F(G)}(u, v) \\
= & \mathscr{A} \mathscr{T} \mathscr{C} \mathscr{I}(G)+\mathscr{A} \mathscr{F} \mathscr{C} \mathscr{I}(G),
\end{aligned}
$$

where $\mathrm{CONN}_{T(G)}(u, v)$ is the $T$-strength of connectedness, and $\operatorname{CONN}_{F(G)}(u, v)$ is the $F$-strength of connectedness between the nodes $u$ and $v$.

Note 3. Obviously, $\mathscr{C} \mathscr{I}(G)$ will not be enhanced by the removal of an edge, and therefore, $\mathscr{A} \mathscr{C} \mathscr{I}(G)$ also. For an IFG, $0 \leq \mathscr{A} \mathscr{C} \mathscr{I}(G) \leq 1$.

Definition 21. Let $G=(N, M)$ be an IFG and $u \in N^{*}$. Then, $u$ is said to be an IF connectivity reducing node (IFCRN) of $G$ if $\mathscr{A} \mathscr{C} \mathscr{I}(G-u)<\mathscr{A} \mathscr{C} \mathscr{I}(G) . u$ is said to be an IF connectivity enhancing node (IFCEN) of $G$ if $\mathscr{A} \mathscr{C} \mathscr{I}(G-u)>\mathscr{A} \mathscr{C} \mathscr{I}(G) . u$ is said to be an IF neutral node of $G$ if $\mathscr{A} \mathscr{C} \mathscr{I}(G-u)=\mathscr{A} \mathscr{C} \mathscr{I}(G)$.

Example 10. Consider the IFG $G$ as shown in Figure 10. We have taken here $\left(T_{N}(u), F_{N}(u)\right)=(0.5,0.5)$ for all $u \in N^{*}$. $\mathscr{A} \mathscr{C} \mathscr{I}(G)=0.16667, \mathscr{A} \mathscr{C} \mathscr{I}\left(G-v_{1}\right)=0.1583, \mathscr{A} \mathscr{C} \mathscr{I}$

$\left(G-v_{2}\right) \quad=0.16667, \mathscr{A} \mathscr{C} \mathscr{I} \quad\left(G-v_{3}\right)=0.1583$, $\mathscr{A} \mathscr{C} \mathscr{I}\left(G-v_{4}\right) \quad=0.1833 . \mathscr{A} \mathscr{C} \mathscr{I}\left(G-v_{1}\right)$, $\mathscr{A} \mathscr{C} \mathscr{I}\left(G-v_{3}\right)<\mathscr{A} \mathscr{C} \mathscr{I} \quad(G), \mathscr{A} \mathscr{C} \mathscr{I}\left(G-v_{2}\right)=\mathscr{A} \mathscr{C} \mathscr{I}(G)$ and $\mathscr{A} \mathscr{C} \mathscr{I}\left(G-v_{4}\right)>\mathscr{A} \mathscr{C} \mathscr{I}(G)$.Thus $v_{1}, v_{3}$ are IFCRNs, $v_{2}$ is an IF neutral node, and $v_{4}$ is a IFCEN. We characterize these nodes using $\mathscr{C} \mathscr{I}$ in the following theorem.

Theorem 5. Let $G=(N, M)$ be an IFG and $u \in N^{*}$ with $\left|N^{*}\right| \geq 3$. Let $r=\mathscr{C} \mathscr{I}(G) / \mathscr{C} \mathscr{I}(G-u)$. $u$ is a IFCEN iff $r<n /(n-2) . u$ is a IFCRN iff $r>n /(n-2) . u$ is an IF neutral node iff $r=n /(n-2)$.

Proof. Suppose that $u$ is an IF neutral node. Then, by definition, $\mathscr{A} \mathscr{C} \mathscr{I}(G-u)=\mathscr{A} \mathscr{C} \mathscr{I}(G)$. By definition of average connectivity index, we obtain

$$
\frac{1}{\left(\begin{array}{l}
n \\
2
\end{array}\right)} \mathscr{C} \mathscr{I}(G)=\frac{1}{\left(\begin{array}{c}
n-1 \\
2
\end{array}\right)} \mathscr{C} \mathscr{I}(G-u) .
$$

From here, we get 


$$
\frac{\mathscr{C} \mathscr{I}(G)}{\mathscr{C} \mathscr{I}(G-u)}=\frac{\left(\begin{array}{c}
n \\
2
\end{array}\right)}{\left(\begin{array}{c}
n-1 \\
2
\end{array}\right)}=\frac{n(n-1) / 2}{(n-1)(n-2) / 2}=\frac{n}{n-2}
$$

The converse part can be proved by reversing the arguments. Similarly, we can prove other cases.

Note 4. An isolated vertex $w$ in an IFG, and $G=(N, M)$ is an IFCEN. Further, if $\bar{G}=(\bar{N}, \bar{M})$ denoje complement of IFG $G=(N, M)$, then $0 \leq \mathscr{C} \mathscr{I}(\bar{G}) \leq\left(\begin{array}{c}n \\ 2\end{array}\right)$ and therefore,

$$
\mathscr{C} \mathscr{F}(G)+\mathscr{C} \mathscr{I}(\bar{G}) \leq\left(\begin{array}{l}
n \\
2
\end{array}\right)+\left(\begin{array}{l}
n \\
2
\end{array}\right)=2\left(\begin{array}{l}
n \\
2
\end{array}\right)=2 \frac{n(n-1)}{2}=n(n-1)
$$

Theorem 6. Let $G=(N, M)$ be an IFG with $\left|N^{*}\right| \geq 3$. If $w \in N^{*}$ is an end vertex of $G$, let $l=\sum_{u \in N^{*}-\{w\}} \operatorname{CONN}_{T(G)}(u, w)+\sum_{u \in N^{*}-\{w\}} \operatorname{CONN}_{F(G)}(u, w)$. Then,

(1) $w$ is an IFCEN if $l<(2 /(n-2)) \mathscr{C} \mathscr{F}(G-w)$

(2) $w$ is an IFCRN if $l>(2 /(n-2)) \mathscr{C} \mathscr{I}(G-w)$
(3) $w$ is an IF neutral node if $l=(2 /(n-2)) \mathscr{C} \mathscr{I}(G-w)$

Proof. Let $w$ be an IF neutral node. Then, by definition, $\mathscr{A} \mathscr{C} \mathscr{I}(G-w)=\mathscr{A} \mathscr{C} \mathscr{F}(G)$. We see that

$$
\begin{aligned}
& \mathscr{C} \mathscr{F}(G)=\mathscr{C} \mathscr{F}(G-w)+\sum_{u \in N^{*}\{w\}} \operatorname{CONN}_{T(G)}(u, w)+\sum_{u \in N^{*}\{w\}} \operatorname{CONN}_{F(G)}(u, w) \\
& \mathscr{C} \mathscr{I}(G)=C I(G-w)+l, \\
& l=\mathscr{C} \mathscr{I}(G)-\mathscr{C} \mathscr{I}(G-w), \\
& \frac{1}{\left(\begin{array}{l}
n \\
2
\end{array}\right)} l=\frac{1}{\left(\begin{array}{l}
n \\
2
\end{array}\right)} \mathscr{C} \mathscr{I}(G)-\frac{1}{\left(\begin{array}{l}
n \\
2
\end{array}\right)} \mathscr{C} \mathscr{I}(G-w) \\
& =\frac{1}{\left(\begin{array}{c}
n-1 \\
2
\end{array}\right)} \mathscr{C} \mathscr{I}(G-w)-\frac{1}{\left(\begin{array}{l}
n \\
2
\end{array}\right)} \mathscr{C} \mathscr{F}(G-w) \\
& =\mathscr{C} \mathscr{I}(G-w)\left[\frac{1}{\left(\begin{array}{c}
n-1 \\
2
\end{array}\right)}-\frac{1}{\left(\begin{array}{l}
n \\
2
\end{array}\right)}\right], \\
& l=\mathscr{C} \mathscr{F}(G-w)\left[\frac{\left(\begin{array}{l}
n \\
2
\end{array}\right)}{\left(\begin{array}{c}
n-1 \\
2
\end{array}\right)}-1\right] \\
& =\frac{2}{n-2} \mathscr{C} \mathscr{I}(G-w) \text {. }
\end{aligned}
$$


The converse part can be proved by reversing these steps. Similarly, we can prove other parts.

At this stage, we can classify an IFG depending on the nature of vertices in it.

Definition 22. An IFG $G$ containing at least one IFCEN is called an IF connectivity enhancing graph. If there is no IFCEN node in $G$ and at least one IFCRN, then $G$ is said to be an IF connectivity reducing graph. If $G$ has all vertices as IF neutral nodes, then it is said to be an IF neutral graph.

\section{Applications}

In this section, two applications are given. One is on Internet routing, and the other is on transport network flow.

7.1. Internet Routing. The strength of connectedness between points in a network has much importance in various areas, for example, shortest path problem, routing problem, network flow problem, and maximum band width problem. Consider a network $G$ that connects routers in a part of a network. For convenience in calculations, we have taken $\left(T_{N}(v), F_{N}(v)\right)=(0.9,0.1)$ for all $v$. Here, the edge values represent maximum bandwidth between the corresponding routers. Membership value of the edge represents correct information and nonmembership value for incorrect information. Also, if $P$ is a path connecting two routers in the network, then $S_{T}(P)$ denotes the truth bandwidth of $P$ and similarly, $S_{F}(P)$ is for falsity bandwidth of $P$. Hence, $\mathrm{CONN}_{T(G)}(u, v)$ and $\mathrm{CONN}_{F(G)}(u, v)$ denote the maximum possible truth bandwidth and the minimum possible falsity bandwidth between $u$ and $v$. Consider the fuzzified network as shown in Figure 11.

After computations, we have $\mathscr{T} \mathscr{C} \mathscr{I}(G)$ $=20.3715, \mathscr{A} \mathscr{T} \mathscr{C} \mathscr{I}(G)=(20.3715 / 55)=0.3704, \mathscr{F} \mathscr{C} \mathscr{I}(G)$ $=0.055, \mathscr{A} \mathscr{F} \mathscr{C} \mathscr{I}(G)=(0.055 / 55)=0.001$. By removal routers $v_{11}$ and $v_{2}$, we obtain $\mathscr{T} \mathscr{C} \mathscr{I}\left(G-v_{11}\right)$ $=15.50745, \mathscr{A} \mathscr{T} \mathscr{C} \mathscr{I} \quad\left(G-v_{11}\right)=(15.50745 \quad / 45)$ $=0.34461, \mathscr{F} \mathscr{C} \mathscr{I}\left(G-v_{11}\right)=0.045, \quad \mathscr{T} \mathscr{C} \mathscr{I}\left(G-v_{11}\right)$ $=15.50745, \mathscr{A} \mathscr{T} \mathscr{C} \mathscr{I}\left(G-v_{11}\right)=(15.50745 / 45)=0.34461$, $\mathscr{F} \mathscr{C} \mathscr{I}\left(G-v_{11}\right)=0.045, \mathscr{A} \mathscr{T} \mathscr{C} \mathscr{I}\left(G-v_{2}\right)=(18.3465 / 45)=$ $0.4077, \mathscr{F} \mathscr{C} \mathscr{I}\left(G-v_{2}\right)=0.045, \mathscr{A} \mathscr{F} \mathscr{C} \mathscr{I}\left(G \quad-v_{2}\right)=0.001$. Hence, $\quad \mathscr{C} \mathscr{I}(G)=\mathscr{T} \mathscr{C} \mathscr{I}(G)+\mathscr{F} \mathscr{C} \mathscr{I}(G)=20.3715$ $+0.055=20.4265, \mathscr{A} \mathscr{C} \mathscr{I}(G)=0.3714, \quad \mathscr{A} \mathscr{C} \mathscr{I}\left(G-v_{11}\right)$ $=0.34561, \mathscr{A} \mathscr{C} \mathscr{I}\left(G-v_{2}\right)=0.4087$. We see that $\mathscr{A} \mathscr{C} \mathscr{I}(G-$ $\left.v_{2}\right)>\mathscr{A} \mathscr{C} \mathscr{I}(G)$ and $\mathscr{A} \mathscr{C} \mathscr{I}\left(G-v_{11}\right)<\mathscr{A} \mathscr{C} \mathscr{I}(G)$. This implies that $v_{2}$ is IFCEN, and $v_{11}$ is a IFCRN.

This problem has less incorrect information because $\mathscr{T} \mathscr{C} \mathscr{I}(G)>\mathscr{F} \mathscr{C} \mathscr{I}(G)$. Also, the average bandwidth of the network is increased by the removal of router $v_{2}$ and removal of $v_{11}$ causes reduction in average bandwidth.

7.2. Transport Network Flow. Consider a directed network $G$ of traffic flow as shown in Figure 12, which is fuzzified. Conveniently, we have taken $\left(T_{N}(v), F_{N}(v)\right)=(0.8,02)$ for all $v \in V(G)$. The connectivity of directed IFG and undirected IFG is similar. So, we can extend these concepts for directed IFG. The vertices are junctions containing correct and incorrect values for vehicles. The edges represent roads connecting two junctions, and their weights indicate number of vehicles consisting of correct and incorrect information. Now, we discuss some connectivity properties of the network flow. Firstly, we find the associated $T$-connectivity matrix TM $(G)$ of the directed IFG.

$$
\operatorname{TM}(\overleftarrow{G})=\left[\begin{array}{ccccc}
0 & 0.4 & 0.4 & 0.4 & 0.6 \\
0.8 & 0 & 0.2 & 0.4 & 0.6 \\
0.8 & 0.8 & 0 & 0.4 & 0.6 \\
0.6 & 0.6 & 0.6 & 0 & 0.6 \\
0.4 & 0.4 & 0.4 & 0.4 & 0
\end{array}\right]
$$

As the graph is directed, the above matrix is not symmetric. So, we need to sum up all the elements of the matrix. Thus, $\mathscr{T} \mathscr{C} \mathscr{I}(G) \quad=6.656, \mathscr{A} \mathscr{T} \mathscr{C} \mathscr{I}(G)=(6.656$ $/ 10) \equiv 0.6656$. Now, the associated $F$ - connectivity matrix $\operatorname{FM}(G)$ is given as follows.

$$
\operatorname{FM}(\stackrel{\leftarrow}{G})=\left[\begin{array}{ccccc}
0 & 0.4 & 0.4 & 0.4 & 0.4 \\
0.2 & 0 & 0.4 & 0.4 & 0.4 \\
0.2 & 0.2 & 0 & 0.4 & 0.4 \\
0.4 & 0.4 & 0.4 & 0 & 0.4 \\
0.4 & 0.4 & 0.4 & 0.4 & 0
\end{array}\right] .
$$

By adding all the entries of $\operatorname{FM}(\overleftarrow{G})$, we obtain $\mathscr{F} \mathscr{C} \mathscr{I}(G)=0.296, \mathscr{A} \mathscr{F} \mathscr{C} \mathscr{I}(G)=(0.296 / 10)=0.0296$.

Thus, $\quad \mathscr{A} \mathscr{C} \mathscr{I}(G)=\mathscr{A} \mathscr{T} \mathscr{C} \mathscr{I}(G)+\mathscr{A} \mathscr{F} \mathscr{C} \mathscr{I}(\overleftarrow{G}$ )$=0.6656+0,0296=0.6952$.

Consider $G-v_{5}$. It is also directed IFG. The matrices $\operatorname{TM}\left(G-v_{5}\right)$ and $\operatorname{TM}\left(G-v_{5}\right)$ are given by

$$
\begin{aligned}
\operatorname{TM}\left(\overleftarrow{G}-v_{5}\right) & =\left[\begin{array}{cccc}
0 & 0.2 & 0.2 & 0.2 \\
0.8 & 0 & 0.2 & 0.2 \\
0.8 & 0.8 & 0 & 0.2 \\
0.6 & 0.6 & 0.6 & 0
\end{array}\right], \\
\operatorname{FM}\left(\overleftarrow{G}-v_{5}\right) & =\left[\begin{array}{cccc}
0 & 0.6 & 0.6 & 0.6 \\
0.2 & 0 & 0.6 & 0.6 \\
0.2 & 0.2 & 0 & 0.6 \\
0.4 & 0.4 & 0.4 & 0
\end{array}\right] .
\end{aligned}
$$

By $\leftarrow \quad$ calculations, $\leftarrow$ we have $\mathscr{T} \mathscr{C} \mathscr{I}\left(G-v_{5}\right)=3.456 \mathscr{A} \mathscr{T} \mathscr{C} \mathscr{I}\left(G-v_{5}\right)=(3.456 / 6)=$ 0.576 and $\mathscr{F} \mathscr{C} \mathscr{I}\left(G-v_{5}\right)=0.216, \mathscr{A} \mathscr{F} \mathscr{C} \mathscr{I} \quad\left(G-v_{5}\right)$ $=(0.216 / 6)=0,036$. Thus, $\mathscr{A} \mathscr{C} \mathscr{I}\left(G-v_{5}\right)=\mathscr{A} \mathscr{T} \mathscr{C} \mathscr{I}(G-$ $\left.v_{5}\right)+\mathscr{A} \mathscr{F} \mathscr{C} \mathscr{I}\left(G-v_{5}\right)=0.576 \quad+0.036=0.612$. As $\mathscr{A} \mathscr{C} \mathscr{I}(G)>\mathscr{A} \mathscr{C} \mathscr{I}\left(G_{\leftleftarrows} v_{5}\right)$, which implies that $v_{5}$ is IFCRN.

Next, we consider $G-v_{1}$. The matrices $\operatorname{TM}\left(G-v_{1}\right)$ and $\operatorname{TM}\left(G-v_{1}\right)$ are given by 


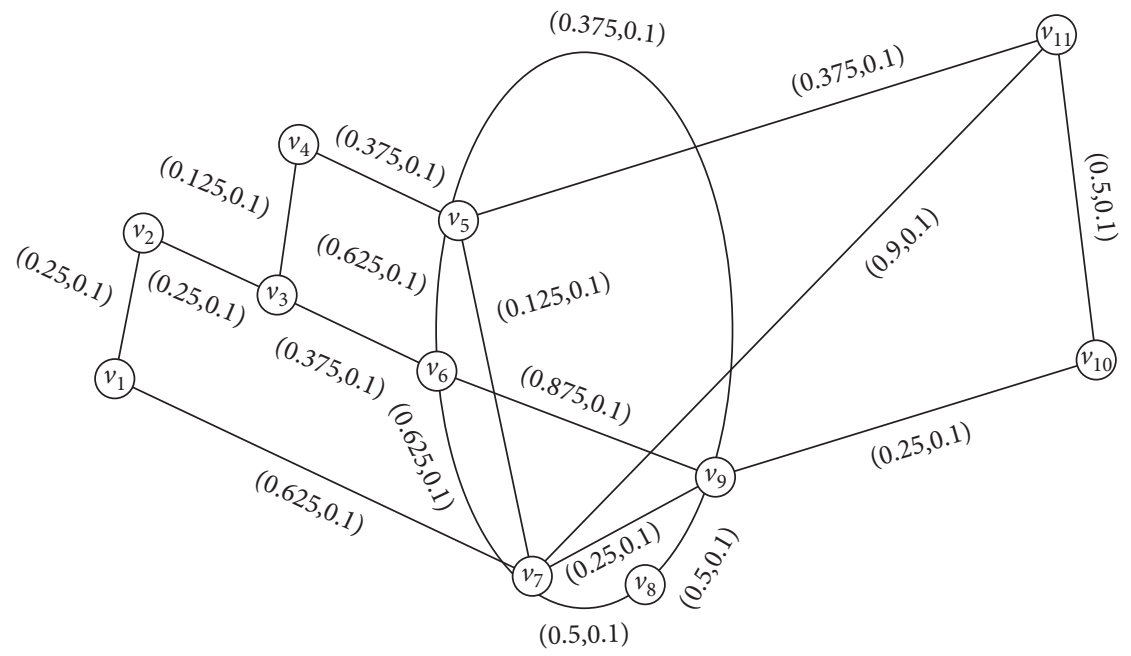

FIGURE 11: Fuzzified Network of routers.

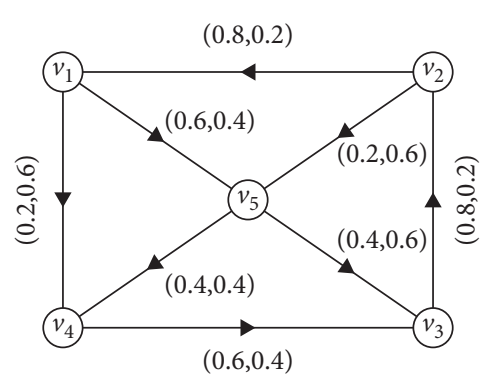

Figure 12: Transport flow network.

TABLE 1: Difference in connectivity indices after removing a vertex.

\begin{tabular}{lcc}
\hline$G-v_{i}$ & $\mathscr{A} \mathscr{C} \mathscr{I}\left(G-v_{i}\right)$ & $\left|\mathscr{A} \mathscr{C} \mathscr{I}(G)-\mathscr{A} \mathscr{C} \mathscr{I}\left(G-v_{i}\right)\right|$ \\
\hline$G-v_{1}$ & 0.508 & 0.1872 \\
$G-v_{2}$ & 0.576 & 0.1192 \\
$G-v_{3}$ & 0.3229 & 0.3723 \\
$G-v_{4}$ & 0.74 & 0.0448 \\
\hline
\end{tabular}

$$
\begin{aligned}
\operatorname{TM}\left(\stackrel{\leftarrow}{G}-v_{1}\right) & =\left[\begin{array}{cccc}
0 & 0.2 & 0.2 & 0.2 \\
0.8 & 0 & 0.2 & 0.2 \\
0.6 & 0.6 & 0 & 0.2 \\
0.4 & 0.4 & 0.4 & 0
\end{array}\right], \\
\operatorname{FM}\left(\stackrel{\leftarrow}{G}-v_{1}\right) & =\left[\begin{array}{cccc}
0 & 0.6 & 0.6 & 0.6 \\
0.2 & 0 & 0.6 & 0.6 \\
0.4 & 0.4 & 0 & 0.6 \\
0.4 & 0.4 & 0.4 & 0
\end{array}\right] .
\end{aligned}
$$

We have ${ }_{\leftarrow}$ after calculations, $\mathscr{T} \mathscr{C} \mathscr{I}\left(\overleftarrow{G}-v_{1}\right)$ $=2.816, \mathscr{A} \mathscr{T} \mathscr{C} \mathscr{I}\left(G-v_{1}\right)=(2.816 / 6)=0.4693 \quad$ and $\mathscr{F} \mathscr{C} \mathscr{I}\left(G-v_{1}\right)=6.232, \mathscr{A} \mathscr{F} \mathscr{C} \mathscr{I}\left(G-v_{k}\right)=(0.232 / 6)=$ 0.0387. Thus, $\mathscr{A} \mathscr{C} \mathscr{I}\left(G-v_{1}\right)=\mathscr{A} \mathscr{T} \mathscr{C} \mathscr{J}\left(G-v_{1}\right)+\mathscr{A} \mathscr{F} \mathscr{C} \mathscr{I}$ $\left(G-v_{1}\right)=0.4693+0.0387=0.508$. As $\mathscr{A} \mathscr{C} \mathscr{I}(G)$ $>\mathscr{A} \mathscr{C} \mathscr{I}\left(G-v_{1}\right)$, which implies that $v_{1}$ is also IFCRN.
Consider $\stackrel{\leftarrow}{G}-v_{2}$. The matrices $\operatorname{TM}\left(\overleftarrow{G}-v_{2}\right)$ and $\operatorname{TM}(\overleftarrow{G}-$ $v_{2}$ ) are given by

$$
\begin{aligned}
\operatorname{TM}\left(\overleftarrow{G}-v_{2}\right) & =\left[\begin{array}{cccc}
0 & 0.4 & 0.4 & 0.6 \\
0 & 0 & 0 & 0 \\
0 & 0.6 & 0 & 0 \\
0 & 0.4 & 0.4 & 0
\end{array}\right], \\
\operatorname{FM}\left(\stackrel{\leftarrow}{G}-v_{2}\right) & =\left[\begin{array}{cccc}
0 & 0.6 & 0.4 & 0.4 \\
0 & 0 & 0 & 0 \\
0 & 0.4 & 0 & 0 \\
0 & 0.4 & 0.4 & 0
\end{array}\right] .
\end{aligned}
$$

$\leftarrow$ We calculate using above matrices $\mathscr{T} \mathscr{C} \mathscr{J}$ $\left(\stackrel{\leftarrow}{G}-v_{2}\right)=1,792, \mathscr{A} \mathscr{T} \mathscr{C} \mathscr{F}\left(G-v_{2}\right)=(1,792 / 6)=0.2987$ and $\mathscr{F} \mathscr{C} \mathscr{I}\left(G-v_{2}\right)=1.664, \mathscr{A} \mathscr{F} \mathscr{C} \mathscr{I}\left(G-v_{2}\right)=(1,664 / 6)$ $=0.2773$. $\leftarrow$ Thus, $\mathscr{A} \mathscr{C} \mathscr{I}\left(G-v_{2}\right)=\mathscr{A} \mathscr{T} \mathscr{C} \mathscr{I}\left(G-v_{2}\right)$ $+\mathscr{A} \mathscr{F} \mathscr{C} \mathscr{I}\left(G_{\leftarrow} v_{2}\right)=0.2987+0.2773=0.576$. As $\mathscr{A} \mathscr{C} \mathscr{I}$ $(G)>\mathscr{A} \mathscr{C} \mathscr{I}\left(G-v_{2}\right)_{2}$ which implies that $v_{2}$ is also IFCRN. Now $_{2}$ we consider $G-v_{3}$. The matrices $\operatorname{TM}\left(G-v_{3}\right)$ and $\operatorname{TM}\left(G-v_{3}\right)$ are given by

$$
\begin{aligned}
& \operatorname{TM}\left(\stackrel{\leftarrow}{G}-v_{3}\right)=\left[\begin{array}{cccc}
0 & 0 & 0.4 & 0.6 \\
0 & 0.4 & 0.6 & \\
0 & 0 & 0 & 0 \\
0 & 0 & 0.4 & 0
\end{array}\right], \\
& \operatorname{FM}\left(\stackrel{\leftarrow}{G}-v_{3}\right)=\left[\begin{array}{cccc}
0 & 0 & 0.4 & 0.4 \\
0.2 & 0 & 0.4 & 0.4 \\
0 & 0 & 0 & 0 \\
0 & 0 & 0.4 & 0
\end{array}\right] .
\end{aligned}
$$

From the above matrices, we have $\mathscr{T} \mathscr{C F}\left(\stackrel{\leftarrow}{G}-v_{3}\right)=$ $1.8, \mathscr{A} \mathscr{T} \mathscr{C} \mathscr{F}\left(G-v_{3}\right)=(1.8 / 6)=0.3$ and $\mathscr{F} \mathscr{C} \mathscr{F}\left(G-v_{3}\right)=$ $0.1375, \mathscr{A} \mathscr{F} \mathscr{C} \mathscr{I}\left(G-v_{3}\right)=\left(\begin{array}{lll}0.1375 & / 6\end{array}\right)=0.0229$. Thus, 
$\mathscr{A} \mathscr{C} \mathscr{I}\left(\overleftarrow{G}-v_{3}\right)=\mathscr{A} \mathscr{T} \mathscr{C} \mathscr{I}\left(\overleftarrow{G}-v_{3}\right)+\mathscr{A} \mathscr{F} \mathscr{C} \mathscr{I}\left(\overleftarrow{G}-v_{3}\right)=$ $0.3+0.0229=0.3229$. As $\mathscr{A} \mathscr{C} \mathscr{I}(G)>\mathscr{A} \mathscr{C} \mathscr{I}\left(G-v_{3}\right)$, which implies that $v_{3}$ is also IFCRN. Finally, we consider $G-v_{4}$. The matrices $\operatorname{TM}\left(G-v_{4}\right)$ and $\operatorname{TM}\left(G-v_{4}\right)$ are given by

$$
\begin{aligned}
\operatorname{TM}\left(\overleftarrow{G}-v_{4}\right) & =\left[\begin{array}{cccc}
0 & 0.4 & 0.4 & 0.6 \\
0.8 & 0 & 0.4 & 0.6 \\
0.8 & 0.8 & 0 & 0.6 \\
0.4 & 0.4 & 0.4 & 0
\end{array}\right], \\
\operatorname{FM}\left(\overleftarrow{G}-v_{4}\right) & =\left[\begin{array}{cccc}
0 & 0.6 & 0.6 & 0.4 \\
0.2 & 0 & 0.6 & 0.4 \\
0.2 & 0.2 & 0 & 0.4 \\
0.6 & 0.6 & 0.6 & 0
\end{array}\right] .
\end{aligned}
$$

From the above matrices, we obtain $\mathscr{T} \mathscr{C} \mathscr{I}\left(\overleftarrow{G}-v_{4}\right)=$ $4.224, \mathscr{A} \mathscr{T} \mathscr{C} \mathscr{I}\left(G-v_{4}\right)=(\underline{4} .224 / 6)=0.704$ and $\mathscr{F} \mathscr{C} \mathscr{I}$ $\left(G-v_{4}\right)=0.216, \mathscr{A} \mathscr{F} \mathscr{C} \mathscr{I}\left(G-v_{4}\right)=(0.216 / 6)=0.036$. Thus, $\quad \mathscr{A} \mathscr{C} \mathscr{I}\left(G-v_{4}\right)=\mathscr{A} \mathscr{T} \mathscr{C} \mathscr{I}\left(G-v_{4}\right)+\mathscr{A} \mathscr{F} \mathscr{C} \mathscr{I}$ $\left(G-v_{4}\right)=0.704+0.036=0.74$. As $\mathscr{A} \mathscr{C} \mathscr{I}(G)<\mathscr{A} \mathscr{C} \mathscr{I}$ $\left(G-v_{4}\right)$, which implies that $v_{4}$ is IFCEN. So, the removal of junction $v_{4}$ increases the average connectivity amongst the other junctions. Table 1 shows that there is a small difference between $\mathscr{A} \mathscr{C} \mathscr{I}(G)$ and $\mathscr{A} \mathscr{C} \mathscr{I}\left(G-v_{4}\right)$. So, the removal of $v_{4}$ has no too much effect on the network.

We also see that the difference between $\mathscr{A} \mathscr{C} \mathscr{I}(\overleftarrow{G})$ and $\mathscr{A} \mathscr{C} \mathscr{I}\left(G-v_{3}\right)$ is highest than other differences, so the removal of $v_{3}$ has maximum negative effects on the connectivity.

\section{Conclusion}

We have developed some $\mathscr{C} \mathscr{I} s$ in the IFGs framework due to the reason that IFGs cover uncertainty and vagueness with the help of two membership grades. Some key results of our study are as follows:

(i) We introduced the notion of $\mathscr{C} \mathscr{I}$ for IFGs and developed results on $\mathscr{C} \mathscr{I}$. Examples are also given to support results of $\mathscr{C} \mathscr{I}$.

(ii) $\mathscr{C} \mathscr{I}$ of edge and vertex deleted IFGs with an example is also given.

(iii) We developed SSC, $\theta$-evaluation of vertices, CC, and $\mathscr{C} \mathscr{I}$ of SC and related results.

(iv) $\mathscr{A} \mathscr{C} \mathscr{I}$ of IFG is defined.

(v) Types of connectivity nodes, namely, CEN, CRN, and neutral node, and results on them are introduced.

(vi) Applications in two types of networks, namely, Internet routing and transport flow network.

In the future, we aim to extend our work to the environment of picture fuzzy graphs [44] and T-spherical fuzzy graphs [45]. We also aim to introduce some other connectivity indices in IFGs and investigate their applications.

\section{Advantages}

The main advantages and characteristics of our study are as follows:

(i) The main feature of our study is to develop the concept of $\mathscr{C} \mathscr{I} s$ under IFGs environment due to the fact that IFGs handle uncertain information with two membership grades.

(ii) IFGs are described by two types of components that is membership and nonmembership, while FGs are characterized by only one component.

(iii) Our findings are the generalization of the results of $\mathscr{C} \mathscr{I} s$ in FGs. For example, if we neglect the second component, then our results are converted into the results of FGs. Thus, the results of $\mathscr{C} \mathscr{I} s$ in FGs become the special case of our results in IFGs.

(iv) Comparatively, our study proves that IFGs would have less loss of information as compared to FGs.

\section{Data Availability}

No data were used to support this study.

\section{Conflicts of Interest}

The authors declare no conflicts of interest about the publication of the research article.

\section{Acknowledgments}

The authors are grateful to the Deanship of Scientific Research, King Saud University for supporting through Vice Deanship of Scientific Research Chairs.

\section{References}

[1] L. A. Zadeh, "Fuzzy sets," Information and Control, vol. 8, no. 3, pp. 338-353, 1965.

[2] M. Gen, Y. Tsujimura, and D. Zheng, "An application of fuzzy set theory to inventory control models," Computers \& Industrial Engineering, vol. 33, no. 3-4, pp. 553-556, 1997.

[3] N. Bhardwaj and P. Sharma, "An advanced uncertainty measure using fuzzy soft sets: application to decision-making problems," Big Data Mining and Analytics, vol. 4, no. 2, pp. 94-103, 2021.

[4] F. Masulli, S. Mitra, and P. Gabriella, Eds., Applications of Fuzzy Sets Theory, 7th International Workshop on Fuzzy Logic and Applications, WILF, Camogli, Italy, 2007.

[5] G. Chen, T. T. Pham, and N. Boustany, "Introduction to fuzzy sets, fuzzy logic, and fuzzy control systems," Applied Mechanics Reviews, vol. 54, no. 6, pp. B102-B103, 2001.

[6] M. Voskoglou, Fuzzy Sets, Fuzzy Logic and Their Applications, MDPI-Multidisciplinary Digital Publishing Institute, Basel, Switzerland, 2020

[7] A. R. Mishra, "An extended fuzzy decision-making framework using hesitant fuzzy sets for the drug selection to treat the mild symptoms of Corona virus Disease 2019 (COVID19)," Applied Soft Computing, vol. 103, Article ID 107155, 2021. 
[8] A. Rosenfeld, "Fuzzy graphs," Fuzzy Sets and Their Applications to Cognitive and Decision Processes, pp. 77-95, Academic Press, Cambridge, MA, USA, 1975.

[9] R. T. Yeh and S. Y. Bang, "Fuzzy Relations, Fuzzy Graphs, and Their Applications to Clustering Analysis," Fuzzy Sets and Their Applications to Cognitive and Decision Processes, Academic Press, Cambridge, MA, USA, pp. 125-149, 1975.

[10] J. N. Mordeson, "Fuzzy line graphs," Pattern Recognition Letters, vol. 14, no. 5, pp. 381-384, 1993.

[11] M. O. Massaâ $€^{\mathrm{TM}}$ deh and N. K. Gharaibeh, "Some properties on fuzzy graphs," Advances in Fuzzy Mathematics, vol. 6, pp. 245-252, 2011.

[12] M. O. Massa'deh, "Some contribution on isomorphic fuzzy graphs," Advances and Applications in Discrete Mathematics, vol. 11, p. 199, 2013.

[13] S. Mathew and M. S. Sunitha, "Types of arcs in a fuzzy graph," Information Sciences, vol. 179, no. 11, pp. 1760-1768, 2009.

[14] K. R. Bhutani and A. Rosenfeld, "Strong arcs in fuzzy graphs," Information Sciences, vol. 152, pp. 319-322, 2003.

[15] K. R. Bhutani and A. Rosenfeld, "Fuzzy end nodes in fuzzy graphs," Information Sciences, vol. 152, pp. 323-326, 2003.

[16] K. R. Bhutani and A. Rosenfeld, "Geodesies in fuzzy graphs," Electronic Notes in Discrete Mathematics, vol. 15, pp. 49-52, 2003.

[17] M. Akram, M. Sarwar, and W. A. Dudek, "Bipolar fuzzy sets and bipolar fuzzy graphs," Graphs for the Analysis of Bipolar Fuzzy Information, Springer, Singapore, pp. 1-80, 2021.

[18] M. Akram, M. Sarwar, and W. A. Dudek, "Energy of bipolar fuzzy graphs," Graphs for the Analysis of Bipolar Fuzzy Information, Springer, Singapore, pp. 309-347, 2021.

[19] N. Jan, L. Zedam, T. Mahmood, and K. Ullah, "Cubic bipolar fuzzy graphs with applications," Journal of Intelligent and Fuzzy Systems, vol. 37, no. 2, pp. 2289-2307, 2019.

[20] K. T. Atanassov, "Intuitionistic fuzzy sets," Fuzzy Sets and Systems, vol. 20, no. 1, pp. 87-96, 1986.

[21] S.-M. Shyi-Ming Chen, "Measures of similarity between vague sets," Fuzzy Sets and Systems, vol. 74, no. 2, pp. 217-223, 1995.

[22] Li Dengfeng and C. Cheng, "â€œNew similarity measures of intuitionistic fuzzy sets and application to pattern recognitions," Pattern Recognition Letters, vol. 23, no. 1-3, pp. 221-225, 2002.

[23] Z. Liang and P. Shi, "Similarity measures on intuitionistic fuzzy sets," Pattern Recognition Letters, vol. 24, no. 15, pp. 2687-2693, 2003.

[24] W.-L. Hung and M.-S. Yang, "Similarity measures of intuitionistic fuzzy sets based on Hausdorff distance," Pattern Recognition Letters, vol. 25, no. 14, pp. 1603-1611, 2004.

[25] E. Szmidt and J. Kacprzyk, "A similarity measure for intuitionistic fuzzy sets and its application in supporting medical diagnostic reasoning," Lecture Notes in Computer Sciences, vol. 3020, 2004.

[26] R. Parvathi and M. G. Karunambigai, "Intuitionistic fuzzy graphs," Computational Intelligence, Theory and Applications, vol. 139, pp. 18-20, 2006.

[27] S. S. Dhavudh and R. Srinivasan, "Intuitionistic fuzzy graphs of second type," Advances in Fuzzy Mathematics, vol. 12, pp. 197-204, 2017.

[28] B. Davvaz, N. Jan, T. Mahmood, and K. Ullah, "Intuitionistic fuzzy graphs of nth type with applications," Journal of Intelligent and Fuzzy Systems, vol. 36, no. 4, pp. 3923-3932, 2019.

[29] M. G. Karunambigai, R. Parvathi, and R. Buvaneswari, “Arcs in intuitionistic fuzzy graphs," Notes on Intuitionistic Fuzzy Sets, vol. 17, pp. 37-47, 2011.
[30] M. G. Karunambigai and O. K. Kalaivani, "Matrix representations of intuitionistic fuzzy graphs," International Journal of Scientific and Research Publications, vol. 6, pp. 520-537, 2016.

[31] S. N. Mishra and A. Pal, "Product of interval valued intuitionistic fuzzy graph," Annals of pure and applied mathematics, vol. 5, pp. 37-46, 2013.

[32] S. N. Mishra and A. Pal, "Regular interval-valued intuitionistic fuzzy graphs," Journal of Informatics and Mathematical Sciences, vol. 9, pp. 609-621, 2017.

[33] A. Fallatah, M. O. Massa'deh, and A. a. M. A. a. Alnaser, "Some contributions on operations and connectivity notations in intuitionistic fuzzy soft graphs," Advances and Applications in Discrete Mathematics, vol. 23, no. 2, pp. 117-138, 2020.

[34] M. A. Asad, W. A. AlZoubi, and M. O. Massa'deh, "Bipolar intuitionistic fuzzy graphs and its matrices," Applied Mathematics \& Information Sciences, vol. 14, no. 2, pp. 205-214, 2020.

[35] M. Akram and N. O. Alshehri, "Intuitionistic fuzzy cycles and intuitionistic fuzzy trees," Science World Journal, vol. 2014, Article ID 305836, 11 pages, 2014.

[36] N. Jan, K. Ullah, T. Mahmood et al., "Some root level modifications in interval valued fuzzy graphs and their generalizations including neutrosophic graphs," Mathematics, vol. 7, no. 1, p. 72, 2019.

[37] N. Yaqoob, M. Gulistan, S. Kadry, and H. A. Wahab, "Complex intuitionistic fuzzy graphs with application in cellular network provider companies," Mathematics, vol. 7, no. 1, p. 35, 2019.

[38] M. Akram, A. Ashraf, and M. Sarwar, "Novel applications of intuitionistic fuzzy digraphs in decision support systems," Science World Journal, vol. 2014, Article ID 904606, 11 pages, 2014.

[39] M. Binu, S. Mathew, and J. Mordeson, "Cyclic connectivity index of fuzzy graphs," IEEE Transactions on Fuzzy Systems, vol. 29, 2020.

[40] S. Poulik and G. Ghorai, "Certain indices of graphs under bipolar fuzzy environment with applications," Soft Computing, vol. 24, pp. 1-13, 2019.

[41] M. Binu, S. Mathew, and J. N. Mordeson, "Connectivity index of a fuzzy graph and its application to human trafficking," Fuzzy Sets and Systems, vol. 360, pp. 117-136, 2019.

[42] M. Binu, S. Mathew, and J. N. Mordeson, "Wiener index of a fuzzy graph and application to illegal immigration networks," Fuzzy Sets and Systems, vol. 384, pp. 132-147, 2020.

[43] M. Akram and B. Davvaz, "Strong intuitionistic fuzzy graphs," Filomat, vol. 26, no. 1, pp. 177-196, 2012.

[44] C. Zuo, A. Pal, and A. Dey, "New concepts of picture fuzzy graphs with application," Mathematics, vol. 7, no. 5, p. 470, 2019.

[45] T. Mahmood, K. Ullah, Q. Khan, and N. Jan, “An approach toward decision-making and medical diagnosis problems using the concept of spherical fuzzy sets," Neural Computing \& Applications, vol. 31, no. 11, pp. 7041-7053, 2019. 